\title{
XMM-Newton campaign on the ultraluminous X-ray source NGC 247 ULX-1: outflows
}

\author{
C. Pinto ${ }^{\oplus,},{ }^{1,2}$ R. Soria, ${ }^{3}$ D. J. Walton ${ }^{\circledR},{ }^{4}$ A. D’Aì, ${ }^{1}$ F. Pintore ${ }^{\oplus},{ }^{1}$ P. Kosec, ${ }^{5}$ W. N. Alston, ${ }^{6}$ F. Fuerst, ${ }^{6}$ \\ M. J. Middleton, ${ }^{7}$ T. P. Roberts, ${ }^{8}$ M. Del Santo, ${ }^{1}$ D. Barret, ${ }^{9}$ E. Ambrosi, ${ }^{1}$ A. Robba, ${ }^{1}$ H. Earnshaw ${ }^{\circledR 10}$ \\ and A. C. Fabian 4 \\ ${ }^{1}$ INAF - IASF Palermo, Via U. La Malfa 153, I-90146 Palermo, Italy \\ ${ }^{2}$ ESTEC/ESA, Keplerlaan 1, NL-2201AZ Noordwijk, the Netherlands \\ ${ }^{3}$ College of Astronomy and Space Sciences, University of the Chinese Academy of Sciences, Beijing 100049, China \\ ${ }^{4}$ Institute of Astronomy, Madingley Road, Cambridge CB3 OHA, UK \\ ${ }^{5}$ MIT Kavli Institute for Astrophysics and Space Research, Cambridge, MA 02139, USA \\ ${ }^{6}$ ESAC/ESA European Space Astronomy Center, PO Box 78, E-28691 Villanueva de la Canada, Madrid, Spain \\ ${ }^{7}$ Physics \& Astronomy, University of Southampton, Southampton, Hampshire SO17 1BJ, UK \\ ${ }^{8}$ Department of Physics, Centre for Extragalactic Astronomy, Durham University, South Road, Durham DH1 3LE, UK \\ ${ }^{9}$ Université de Toulouse, CNRS, IRAP, 9 Avenue du colonel Roche, BP 44346, F-31028 Toulouse Cedex 4, France \\ ${ }^{10}$ Cahill Center for Astronomy and Astrophysics, California Institute of Technology, Pasadena, CA 91125, USA
}

Accepted 2021 June 3. Received 2021 May 28; in original form 2021 April 22

\begin{abstract}
ABSTRA CT
Most ultraluminous X-ray sources (ULXs) are believed to be powered by super-Eddington accreting neutron stars and, perhaps, black holes. Above the Eddington rate the disc is expected to thicken and to launch powerful winds through radiation pressure. Winds have been recently discovered in several ULXs. However, it is yet unclear whether the thickening of the disc or the wind variability causes the switch between the classical soft and supersoft states observed in some ULXs. In order to understand such phenomenology and the overall super-Eddington mechanism, we undertook a large (800 ks) observing campaign with XMM-Newton to study NGC 247 ULX-1, which shifts between a supersoft and classical soft ULX state. The new observations show unambiguous evidence of a wind in the form of emission and absorption lines from highly ionized ionic species, with the latter indicating a mildly relativistic outflow $(-0.17 c)$ in line with the detections in other ULXs. Strong dipping activity is observed in the light curve and primarily during the brightest observations, which is typical among soft ULXs, and indicates a close relationship between the accretion rate and the appearance of the dips. The latter is likely due to a thickening of the disc scale height and the wind as shown by a progressively increasing blueshift in the spectral lines.
\end{abstract}

Key words: accretion, accretion discs - X-rays: binaries - X-rays: individual: NGC 247 ULX-1.

\section{INTRODUCTION}

There is a consensus that the majority of ultraluminous X-ray sources (ULXs) are stellar-mass compact objects (neutron stars and perhaps black holes) accreting above the critical Eddington rate (see, e.g. King et al. 2001; Poutanen et al. 2007; Middleton et al. 2011; Bachetti et al. 2014; Kaaret, Feng \& Roberts 2017). The spectral curvature in the X-ray band, with a characteristic downturn above $\sim 5 \mathrm{keV}$, and the presence of residuals at energies $\lesssim 1 \mathrm{keV}$ when modelled with featureless continuum models, are two characteristic ULX features (see, e.g. Soria et al. 2004; Goad et al. 2006; Gladstone, Roberts \& Done 2009) that are naturally explained if the primary X-ray photons are seen through a disc wind, as expected for systems accreting in the super-Eddington regime (e.g. Shakura \& Sunyaev 1973; Poutanen et al. 2007). The thickness of the wind and, as a result, the 'softness' of the observed ULX spectra in the $\sim 0.1-20 \mathrm{keV}$ band, are likely

^E-mail: ciropinto1982@gmail.com a function of two main parameters: the mass outflow rate in the wind (which is related to the accretion rate), and our viewing angle, with softer sources being observed closer to the disc plane (see, e.g. Sutton, Roberts \& Middleton 2013; Middleton et al. 2015a; Pinto et al. 2017). In particular, ULXs with a power-law photon index $\Gamma$ $>2$ in the $\sim 0.3-5 \mathrm{keV}$ band are empirically classified in a 'soft ultraluminous' (SUL) state.

\subsection{Supersoft ultraluminous sources}

Among the ULXs, a special subclass is represented by ultraluminous supersoft sources (ULSs), also referred to as sources in the 'supersoft ultraluminous' (SSUL) state (Feng et al. 2016). This state is defined by a dominant, cool blackbody component $\left(T_{\mathrm{bb}} \lesssim 140 \mathrm{eV}\right)$, with very weak or completely absent hard component at higher energies. Observationally, sources in the SSUL state show essentially no photons $>1 \mathrm{keV}$. Their bolometric luminosity is typically a few $10^{39} \mathrm{erg} \mathrm{s}^{-1}$. They should not be confused with the 'classical' supersoft sources (see, e.g. Krautter et al. 1996), which are usually 
interpreted as nuclear burning on the surface of a white dwarf. Classical supersoft sources also have blackbody spectra, but at lower luminosities $\left(L_{\mathrm{bb}} \lesssim 10^{38} \mathrm{erg} \mathrm{s}^{-1}\right.$ ), and generally with a smaller bb radius: $R_{\mathrm{bb}} \sim 5000-10000 \mathrm{~km}$, consistent with a white dwarf, while SSUL spectra can have blackbody radii as large as $10^{5} \mathrm{~km}$ (Urquhart \& Soria 2016).

Blackbody modelling of SSUL spectra at different epochs shows that the characteristic radius is not constant, which rules out a hard surface as the origin of the thermal emission (Feng et al. 2016). It also shows a clear anticorrelation between blackbody radius and temperature, which rules out a standard accretion disc. Instead, such behaviour is consistent with emission from the photosphere of an optically thick wind. The increase in the photospheric radius corresponds to an enhancement of the wind thickness.

The apparent non-periodic spectral variability in the SSUL state (Liu 2008) is very likely due to a thickening of the wind along our line of sight. One question we want to address is whether the shortterm variations in optical depth are just stochastic variability (at a given mass accretion rate $\dot{M}$ ) due to the clumpy nature of the wind ('weather', see, e.g. Takeuchi, Ohsuga \& Mineshige 2013), or instead each variation is driven by a change in the underlying $\dot{M}$ ('climate'), which then affects the wind density and launching radius.

A deep absorption edge was detected at $\approx 1.0-1.1 \mathrm{keV}$ in several SSUL sources (e.g. those in M 51, NGC 6946 and M 101: Urquhart \& Soria 2016; Earnshaw \& Roberts 2017). This feature clearly appears when the source softens, progressively losing all X-ray photons with energy above $1 \mathrm{keV}$. There are no strong absorption edges predicted at the observed energy of those dips. Viable solutions are blueshifted $\mathrm{O}$ VIII ionization edges ( $871 \mathrm{eV}$ at rest), which was observed in novae during their supersoft phase (e.g. Pinto et al. 2012), or a combination of high-ionization Fe L or Ne IX-X absorption lines with velocities of $\sim 0.1-0.2 \mathrm{c}$, supporting the case for an optically thick, mildly relativistic wind.

\subsection{NGC 247 ULX-1}

Previous X-ray studies (see, e.g. Feng et al. 2016), based on two short XMM-Newton observations, showed that NGC 247 ULX-1 switched from a supersoft state with hardly any flux above $1 \mathrm{keV}$ (in 2009) to a much brighter state (in 2014) consistent with the soft end of the 'standard' ULX population (i.e. those with a bright hard X-ray spectral tail).

NGC 247 ULX-1 is also among the most variable ULXs. It exhibits strong dips in its X-ray light curve, which last several ks, and during which the flux decreases by an order of magnitude (Feng et al. 2016). Both the low and high flux spectra are characterized by strong spectral features.

Pinto et al. (2017) found similarities between the narrow X-ray spectral features of NGC 247 ULX-1 in the high flux state with those of NGC 55 ULX, using observations taken with the high-resolution gratings aboard XMM-Newton. Despite the short duration (33 ks) of the observations available, two remarkable absorption features at 7.5 and $16.2 \AA$ were found along with other weaker features, which can be modelled with absorption from photoionized gas outflowing at $\approx 0.14 c$. The observation taken in the low-flux (supersoft) state is far too short to provide any useful data. Thus, we proposed and were awarded an $800 \mathrm{ks}$ deep XMM-Newton programme to characterize the properties of the outflows and the origin of the spectral residuals, and correlate them with the continuum flux variability and state changes.

This paper is the first in a series of intriguing results from our XMM-Newton campaign. Here, we focus on the search for wind signatures in the time-average spectrum, taking full advantage of the
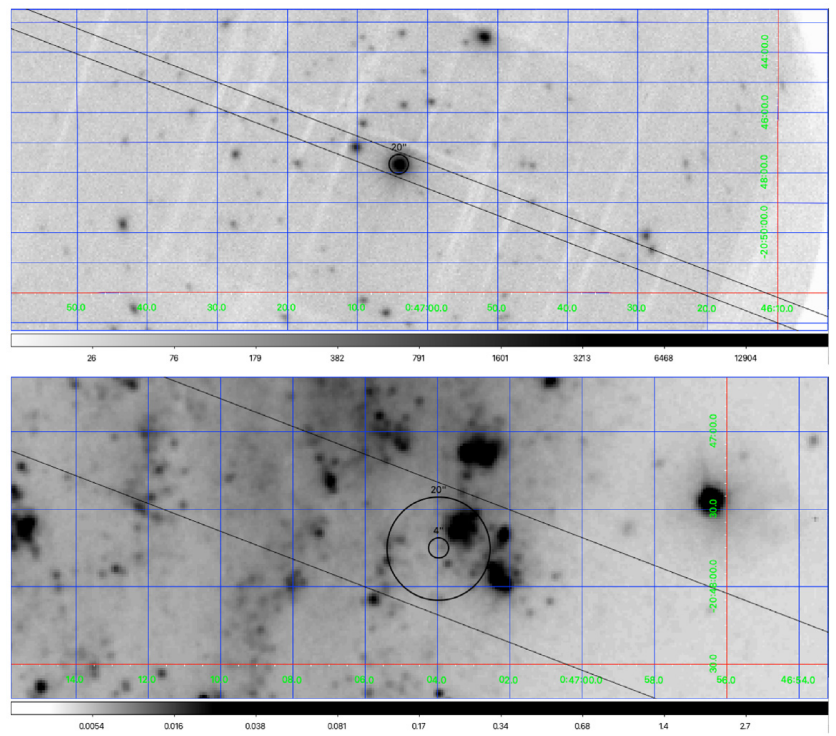

Figure 1. XMM-Newton image of the NGC 247 field obtained by combining all the data available for EPIC-pn and MOS 1,2 zooming on to the ULX-1 region (top panel). The black strip and circle indicate the RGS and EPIC extraction regions, respectively. The bottom panel shows the time-averaged image obtained by stacking all the data from the Optical Monitor. A small circle with 4 arcsec radius shows the X-ray source centroid.

Table 1. XMM-Newton campaign on NGC 247 ULX-1.

\begin{tabular}{lcrrrrr}
\hline OBS_ID & Date & $\begin{array}{c}t_{\text {RGS1 }} \\
(\mathrm{ks})\end{array}$ & $\begin{array}{c}t_{\mathrm{RGS} 2} \\
(\mathrm{ks})\end{array}$ & $\begin{array}{c}t_{\mathrm{MOS} 1} \\
(\mathrm{ks})\end{array}$ & $\begin{array}{c}t_{\mathrm{MOS} 2} \\
(\mathrm{ks})\end{array}$ & $\begin{array}{c}t_{\mathrm{pn}} \\
(\mathrm{ks})\end{array}$ \\
\hline 0844860101 & $2019-12-03$ & 110.5 & 110.1 & 104.6 & 105.0 & 76.6 \\
0844860201 & $2019-12-09$ & 110.9 & 110.6 & 109.3 & 109.2 & 90.3 \\
0844860301 & $2019-12-31$ & 117.4 & 117.0 & 112.6 & 113.9 & 76.8 \\
0844860401 & $2020-01-02$ & 112.3 & 112.0 & 110.6 & 110.5 & 93.5 \\
0844860501 & $2020-01-04$ & 115.9 & 115.6 & 113.3 & 113.3 & 94.4 \\
0844860601 & $2020-01-06$ & 102.3 & 101.7 & 83.3 & 83.2 & 57.4 \\
0844860701 & $2020-01-08$ & 28.2 & 28.3 & 96.1 & 97.5 & 70.1 \\
0844860801 & $2020-01-12$ & 61.0 & 60.8 & 59.3 & 59.4 & 41.7 \\
& & 758.5 & 756.1 & 789.1 & 792.0 & 600.8 \\
Total [ks] & & 7.2 & 10.4 & 56.7 & 57.2 & 186.5 \\
Total [kcnts] & & & & &
\end{tabular}

Note. Exposure times account for high background removal. Source counts are in the whole energy band for each detector.

high-spectral-resolution data, and on the variability of the spectral features around $1 \mathrm{keV}$. We detail our observing campaign in Section 2 and present the results of our spectral analysis in Section 3. We discuss the results in Section 4, and outline some conclusions in Section 5.

\section{NGC 247 XMM-Newton CAMPAIGN}

We observed the NGC 247 galaxy between December 2019 and January 2020. The roll angle was similar throughout the whole campaign and avoided strong contamination along the dispersed grating spectra from the nearby brightest X-ray sources (see Fig. 1 and Appendix A1 for more detail). Seven observations were expected to occur but, owing to an issue with the RGS instrument that occurred during the last observation (id:0844860701), an additional final observation was taken shortly afterwards (id:0844860801) to recover the lost exposure. In Table 1, we report the detail of our observations. We performed the spectral analysis with the SPEX code (Kaastra, Mewe \& Nieuwenhuijzen 1996), we used $C$-statistics ( $C$-stat; Cash 1979) for spectral fits, which was proved to be efficient in comparing 

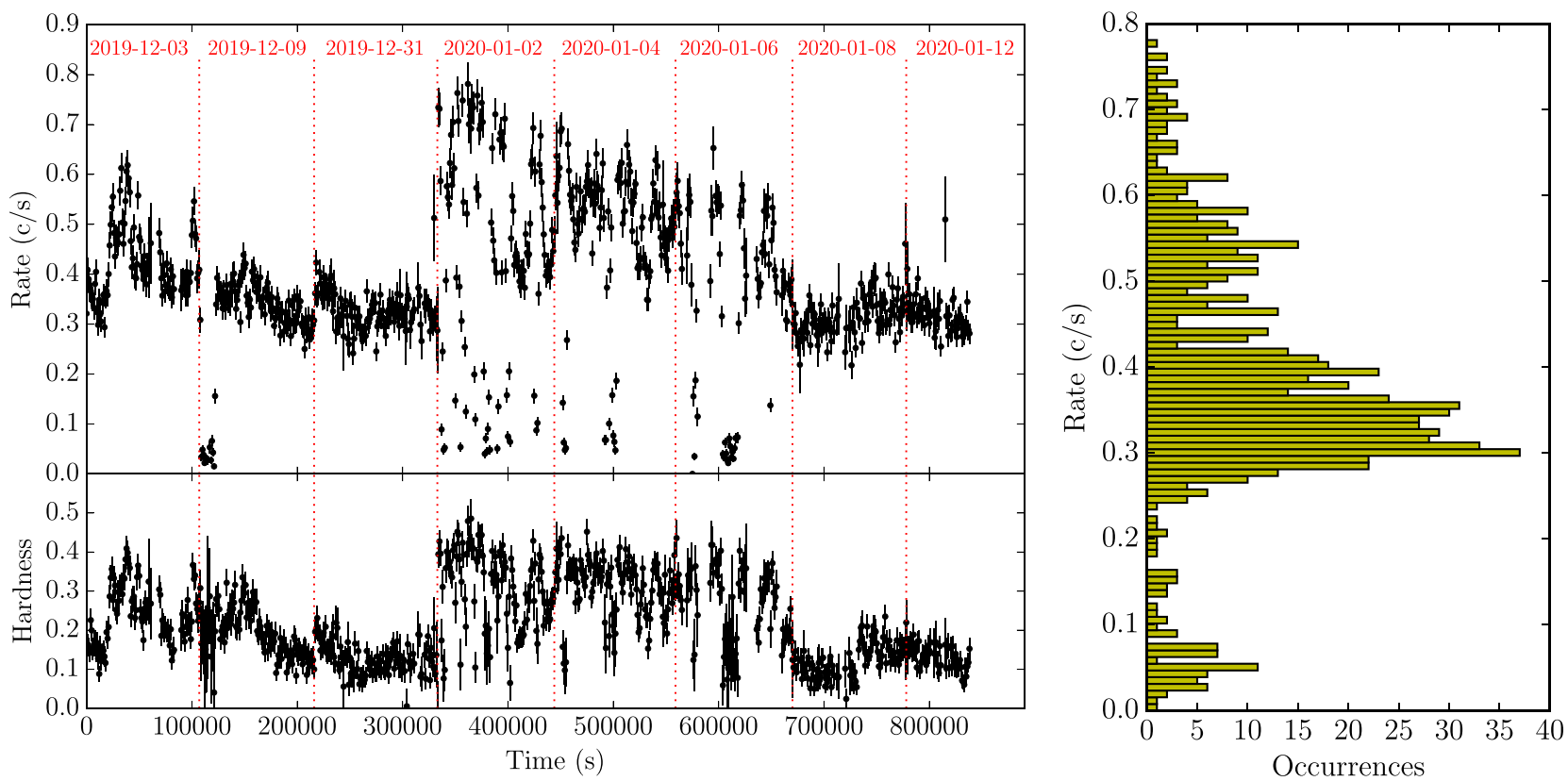

Figure 2. Top left panel: cumulated XMM-Newton $(0.3-10 \mathrm{keV})$ pn light curve of NGC 247 ULX-1 with the individual observations separated by vertical dotted lines. Time bin size is $1 \mathrm{ks}$. Obsid 3-to-7 are separated by $20-60 \mathrm{ks}$ each. Bottom left panel: hardness ratio $(H /(H+S))$ estimated from the light curves in the soft $(0.3-1 \mathrm{keV})$ and hard (1-10 keV) X-ray bands. Right-hand panel: count rate histogram.

models similarly to $\chi^{2}$ statistics (Kaastra 2017), and we adopted $1 \sigma$ confidence intervals.

\subsection{Data preparation}

In this work, we used data from the European Photon-Imaging Camera (EPIC), the Reflection Grating Spectrometer (RGS), and the Optical Monitor (OM) aboard XMM-Newton. The primary science was carried out with the RGS that can detect and resolve narrow spectral features. The broad-band cameras (EPIC MOS 1,2 and pn) were mainly used to determine the spectral continuum and cover the hard X-ray band missed by the RGS.

\subsubsection{EPIC cameras}

We reduced the eight new XMM-Newton observations with the Science Analysis System (SAS v18.0.0, CALDB available on March 2020). EPIC-pn and MOS data were reduced with the EPPROC and EMPROC tasks, respectively. Following the recommended procedures, we filtered the MOS and pn event lists for bad pixels, cosmic ray events outside the field of view, photons in the gaps $(\mathrm{FLAG}=0)$, and applied standard grade selections (PATTERN $\leq 12$ for MOS and PATTERN $\leq 4$ for $\mathrm{pn}$ ). We corrected for contamination from high background by selecting background-quiescent intervals on the light curves for MOS 1,2 and pn in the 10-12 keV energy band. These light curves were extracted in time bins of $100 \mathrm{~s}$ and all those with a count rate above $0.4 \mathrm{c} \mathrm{s}^{-1}$ for pn and $0.2 \mathrm{c} \mathrm{s}^{-1}$ for MOS were rejected. The MOS 1-2 and pn clean exposure times are reported in Table 1.

We extracted EPIC MOS 1-2 and pn images in the $0.3-10 \mathrm{keV}$ energy range and stacked them with the EMOSAIC task (see Fig. 1). We also extracted EPIC MOS 1-2 and pn light curves from within a circular region of $20^{\prime \prime}$ radius centred on the source position $(\alpha$, $\delta=00: 47: 04.0,-20: 47: 45.7)$. We used the task EPICLCCORR, which corrects for vignetting, bad pixels, chip gaps, PSF, and quantum efficiency. The background (BKG) light curves were extracted from within a larger circle in a nearby region on the same chip. In order to measure the variations in the spectra hardness, we extracted EPIC-pn (for its larger effective area) light curves in the soft $(0.3-1 \mathrm{keV})$ and hard (1-10 keV) energy bands (with $1 \mathrm{ks}$ time bins to increase the signal-to-noise ratio of each bin). The PN light curves are shown in Fig. 2 (top-left panel) with a length above $800 \mathrm{ks}$ (despite an effective clean exposure of $600 \mathrm{ks}$ ) due to the large $1 \mathrm{ks}$ bin size which does not show the time gaps of high BKG lasting few 100s. The light curves were also glued together for displaying purposes but with vertical dotted lines separating them. The hardness ratio was defined as the fraction of hard photons with respect to the total $(H /(H+S))$. The boundary energy was adopted owing to the strong spectral curvature of supersoft sources exhibited above $1 \mathrm{keV}$ (see Section 2.1.4).

We extracted EPIC MOS 1-2 and pn spectra in the same regions used for the light curves. The EPIC-pn spectra of the individual observations are shown in Fig. 3. We avoided over-plotting the MOS spectra for displaying purposes.

\subsubsection{RGS cameras}

The RGS data reduction was performed with the RGSPROC pipeline. We filtered out periods affected by contamination from Solar flares by selecting background-quiescent intervals in the light curves of the RGS 1,2 CCD 9 (i.e. $\gtrsim 1.7 \mathrm{keV}$ ) with a count rate below $0.2 \mathrm{c} / \mathrm{s}$. As usual, Solar flares affected the RGS data on a much lower level than EPIC. The total clean exposure times are quoted in Table 1.

We extracted the first and secondorder RGS spectra in a crossdispersion region of $0.8 \mathrm{arcmin}$ width, centred on the source coordinates and the background spectra by selecting photons beyond the 98 per cent of the source point-spread function. The background regions do not overlap with bright sources. After inspecting the second-order spectra, we decided not to use them as they were 

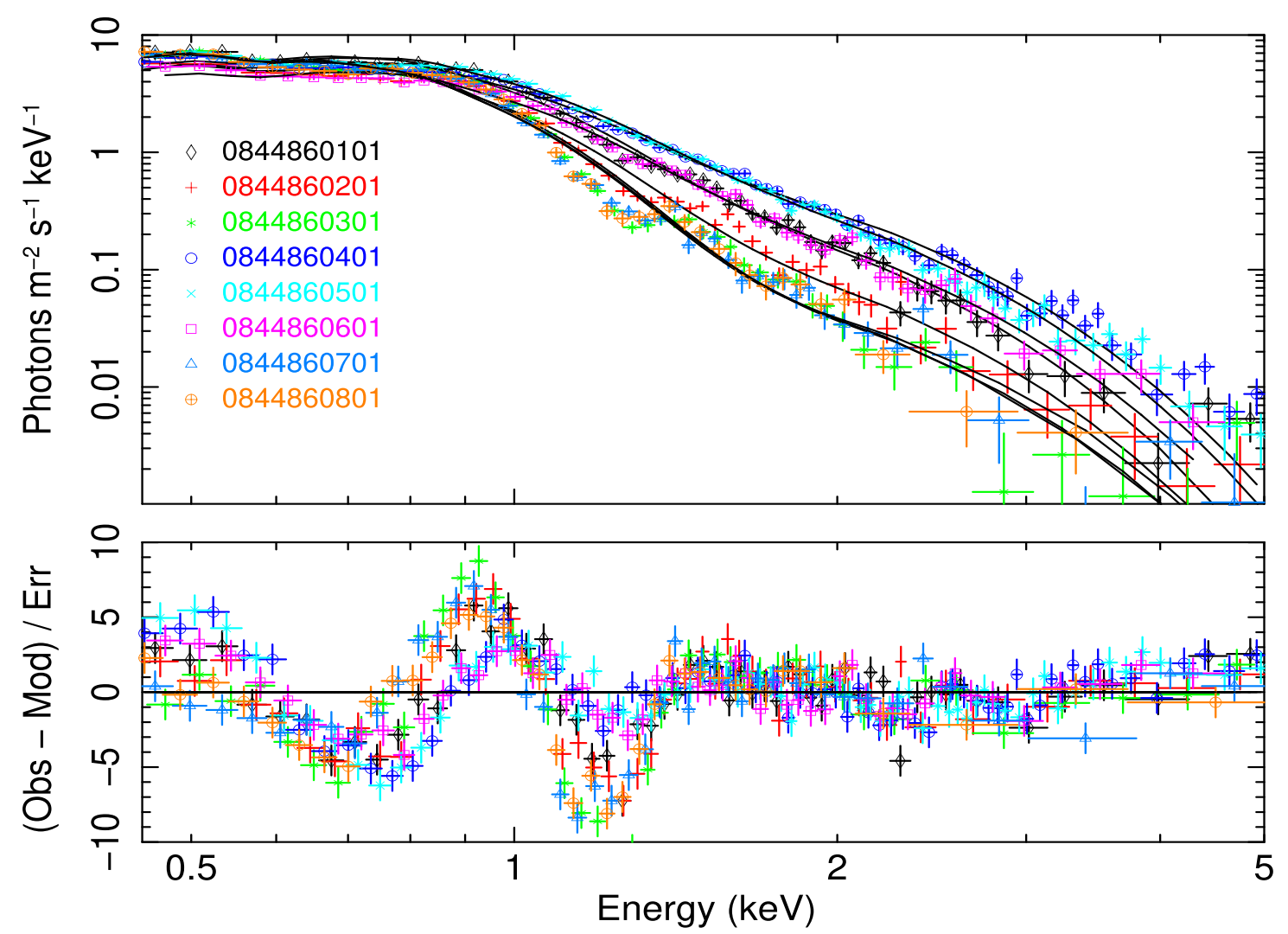

Figure 3. XMM-Newton EPIC-pn spectra of NGC 247 ULX-1. The top panel shows the pn spectra of the individual observations with overlaid the two-blackbody continuum models. The bottom panel shows the corresponding residuals. Both the emission feature below $1 \mathrm{keV}$ and the absorption feature above $1 \mathrm{keV}$ vary in centroid and strength according to the continuum flux and shape. This is qualitatively similar to the intermediate-hard source NGC 1313 ULX-1 and the soft source NGC 55 ULX-1 (Middleton et al. 2015b; Pinto et al. 2017).

highly affected by the background. We stacked the first-order RGS 1 and 2 spectra from all observations with RGSCOMBINE and the EPIC-pn, MOS 1, and MOS 2 spectra, using EPICSPECCOMBINE. The stacking provided 4 time-averaged high signal-to-noise spectra for RGS, MOS 1, MOS 2, and pn detectors.

All XMM-Newton spectra were grouped in channels of at least $1 / 3$ of the spectral resolution, for optimal binning and to avoid oversampling, and at least 25 counts per bin, using SAS task SPECGROUP. This has also the advantage to smooth the background spectra in the energy range with low statistics, avoiding narrow spurious features introduced by the background subtraction. This also enabled us to check our results with the $\chi^{2}$ statistics. The stacked spectra have many counts with the binning affecting only the spectral range at the rather low and high energies (outside the 0.6-1.7 keV RGS band and above $4 \mathrm{keV}$ in EPIC spectra), where line detection is not crucial. We found no significant effect on to our line or continuum modelling by decreasing the binning to just $1 / 3$ of the spectral resolution.

\subsubsection{Optical Monitor}

We used OM data to search for a possible optical/UV counterpart to NGC 247 ULX-1. To increase the signal-to-noise ratio, we stacked all the internally aligned full-frame sky images per filter and per observation, using the SAS tool OMMOSAIC. Each observation contains at least one image in one of these filters: V, UVW1, UM2, and UVW2 and the final total exposures corresponded to 105,105 ,
115 , and $220 \mathrm{ks}$, respectively. We ran the OMDETECT task on these stacked images with a limit on the detection threshold of $2 \sigma$. At the position of the ULX (Section 2.1.1), no source was detected in any of the filters. This is unsurprising since the ULX region is very close to a bright association of $\mathrm{OB}$ stars, which makes such a detection challenging. To derive an upper limit for the ULX emission in these bands, we computed the total background rate for a circular region of 6 arcsec around the position of the ULX. This provided $3 \sigma$ upper limits for the ULX flux, which are comparable to previous measurements obtained by Feng et al. (2016) using deep observations with Hubble Space Telescope (HST, UV-optical fluxes $\sim 10^{-14} \mathrm{erg} \mathrm{s}^{-1} \mathrm{~cm}^{-2}$ ). In the far-UV, the OM flux upper limits are slightly below the HST detections, suggesting long-term flux variability and that a substantial fraction of the UV flux originates within the accretion disc rather than the stellar companion (see Fig. A3, top panel).

A final OM image obtained by stacking the data from all the filters of all the observations is shown in Fig. 1, bottom panel, where the bright association of OB stars can be seen on the right side of the $\mathrm{X}$-ray source centroid.

\subsubsection{Data investigation}

The XMM-Newton $0.3-10 \mathrm{keV}$ light curve shows a strong dipping behaviour with the source flux approaching zero c/s during time interval of less than $15 \mathrm{ks}$, as found by Feng et al. (2016), see Fig. 2 (top-left panel). The dips have variable duration, with the shortest 
ones being of a few hundred seconds, which was estimated extracting finer light curves (D'Aì et al. in preparation). During the dips, the flux drops by an order of magnitude and then returns to the previous level, which makes it difficult to believe it is due to an intrinsic flux change rather than to a temporary obscuration phenomenon. This behaviour causes the multiple peaks present in the histogram of the count rates (Fig. 2, right-hand panel), which disagree with a single-peaked lognormal trend.

The hardness ratio decreases during the dips (Fig. 2, bottom-left panel), which is very similar to the soft source NGC 55 ULX-1 (Pinto et al. 2017). This was interpreted as evidence of temporary obscuration of the inner hot regions from a clumpy disc wind (e.g. Stobbart, Roberts \& Wilms 2006).

The light curve also shows that NGC 247 ULX-1 undergoes a longterm flux variability with observations 4-to-6 exhibiting significantly higher fluxes. Importantly, the frequency of the dips was higher during these observations.

In order to evaluate the source variability during each observation, we calculated the fractional excess variance of the EPIC-pn light curve of each observation and the root mean square (RMS), following standard formulae (see, e.g. Nandra et al. 1997; Vaughan et al. 2003, and Allevato et al. 2013). We adopted time bins of $1 \mathrm{ks}$ and, as time length, the duration of each exposure $(\sim 100 \mathrm{ks}$, with the exception of obsid:0844860801). The computed RMS values are reported in Table A1 and range from about 10 to 55 percent.

The EPIC spectra of the individual observations in Fig. 3 show a variability pattern that is common to ULXs, with the harder band $(>1 \mathrm{keV}$ ) exhibiting the largest variation (see, e.g. Middleton et al. 2015a; Brightman et al. 2016; Walton et al. 2018c). A thorough analysis of the variability pattern involving a careful sampling of time interval with similar flux and hardness ratio, and the study of the power density spectra, will be done in two separate papers (D'Aì et al. in preparation; Alston et al. 2021).

\section{SPECTRAL ANALYSIS}

In this section we present the spectral analysis of NGC 247 ULX1. We first show the time evolution of the main spectral residuals around $1 \mathrm{keV}$ through the modelling of the spectral continuum in EPIC spectra from different observations (see Section 3.1). Then we will perform a thorough analysis of the high-statistics, timeaveraged, stacked spectrum in order to identify the lines in the RGS (Sections 3.2 and 3.3), to build the spectral energy distribution (SED), and to use physical plasma models for the wind detection and modelling (Sections 3.4 and 3.5). The final best-fitting models are shown in Section 3.6 and the statistical significance of our findings in Appendix A5.

ULX spectra require up to three components to obtain a satisfactory description of the spectral continuum. Two blackbody-like components are often used to model the soft $(0.3-1 \mathrm{keV})$ and hard (1-10 keV) X-ray energy bands (see, e.g. Stobbart et al. 2006; Pintore et al. 2015). The availability of high-statistics spectra and broad-band data reveals the presence of a third harder component, which dominates the continuum above $10 \mathrm{keV}$ (see, e.g. Walton et al. 2018a). In the framework of super-Eddington accretion, the cooler, soft, component corresponds to the X-ray emission of the wind and the disc around the spherization radius. The hard component refers to the inner accretion flow. The hard $(>10 \mathrm{keV})$ tail is either due to Compton scattering in the innermost regions or from an accretion column (see, e.g. Middleton et al. 2015a). The two hard components, especially the hard tail, are weak in supersoft ULXs.

\subsection{Time evolution of the $\sim 1 \mathrm{keV}$ residuals}

For the modelling of EPIC spectra of individual observations we adopted a simple continuum model consisting of two blackbody (bb) components, which is often used as a proxy for more complex models (see, e.g. Walton et al. 2014; Pinto et al. 2017; Koliopanos et al. 2017; Gúrpide et al. 2021). We did not model the hard tail in the individual spectra because it is so weak that any model would be highly unconstrained, but for the time-averaged SED modelling we took it into account (see Section 3.2). The emission components are corrected for absorption by the Galactic interstellar medium and the circumstellar medium near the ULX using the hot model in SPEX with a low temperature $(k T=0.2 \mathrm{eV}$, e.g. Pinto et al. 2013), at which the gas is neutral. In the spectral fits, we coupled the column density of the hot model across all observations as it is unlikely that the amount of neutral gas along our line of sight towards the ULX would change on time-scales of a few days. In some Galactic X-ray binaries (XRB) variable obscuration was found, but it is not clear whether these findings are related to ionized gas (i.e. winds) or uncertainties in the continuum rather than neutral gas (see, e.g. Miller, Cackett \& Reis 2009; Walton et al. 2017).

We simultaneously applied the hot $(b b+b b)$ continuum model to the EPIC MOS 1,2 and pn spectra of all eight observations. The results are shown in Fig. 3 and Table A1. We obtained an average column density of $N_{\mathrm{H}}=(3.4 \pm 0.1) \times 10^{21} \mathrm{~cm}^{-2}$. This phenomenological model provides a good description of the broadband spectra, but the $C$-statistics are high when compared to the corresponding degrees of freedom $\left(C_{v} \sim 3-9\right)$ due to the well-known strong and sharp residuals in the form of absorption and emission features around $1 \mathrm{keV}$ (see Fig. 3).

It is possible to understand the nature of the residuals by tracking their temporal evolution from one observation to another. In Middleton et al. (2015b) and Pinto et al. (2017), the three main spectral features observed around $1 \mathrm{keV}$ in the spectra of NGC 1313 ULX1 and NGC 55 ULX-1 were modelled with a positive (emission) and two negative Gaussians (absorption) lines. The availability of deeper observations allow us to fit the three Gaussian components independently, but we fixed the line broadening to zero $\mathrm{km} \mathrm{s}^{-1}$ (i.e. only instrumental broadening) in order to minimize the degeneracy that can be produced by the low spectral resolution of EPIC. We chose three Gaussian lines as previous work on high-resolution RGS data identified $\mathrm{Fe} / \mathrm{Ne}$ emission lines at $1 \mathrm{keV}$, O VIII absorption lines around $0.7-0.8 \mathrm{keV}$, and $\mathrm{Fe} / \mathrm{Ne}$ absorption lines above $1 \mathrm{keV}$ (Pinto, Middleton \& Fabian 2016). Using only one or two Gaussian lines always resulted in significantly worse fits during alternative tests.

The hot $(b b+b b)+($ gaus + gaus + gaus $)$ spectral model improves the fits for all observations with respect to the hot $(b b+b b)$ model. In Table A1, we report the best-fitting parameters for each observation. The reduced $\mathrm{C}$-stat is still rather high due to additional residuals that require more complex and physical models. Moreover there is evidence of a weak, broad, hard tail in all spectra above $3 \mathrm{keV}$, which cannot be explained with atomic lines (Fig. 3).

In Fig. 4, we compare the energy centroids (left-hand panel) and the fluxes (right-hand panel) as measured for the three Gaussian lines in the EPIC spectra of the eight observations. The point size was coded according to the value of their RMS estimate in Section 2.1.4. Both the energies and fluxes of the Gaussian lines vary with the time, showing a higher blueshift and lower flux (in absolute value) during the dipping observations with enhanced variability. 

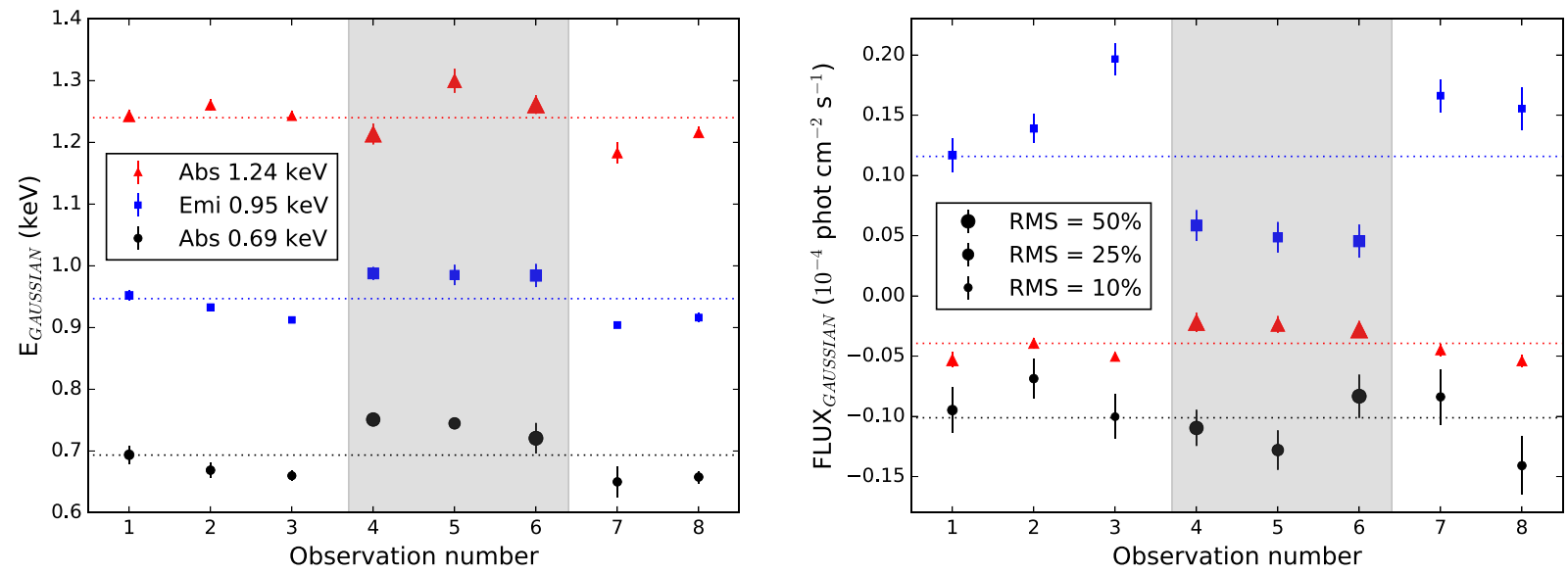

Figure 4. Evolution of the XMM-Newton EPIC spectral residuals around $1 \mathrm{keV}$. The left- and the right-hand panels show the energy centroid and normalization of the Gaussian lines used to fit the three main (unresolved) spectral residuals, respectively. The shaded grey areas highlight the observations of high spectral variability and dipping. 'Abs' and 'Emi' refer to absorption and emission features, respectively.

Table 2. Time-averaged EPIC + RGS spectral fits.

\begin{tabular}{lccc}
\hline Parameter & Units & hot $(b b+b b)$ & hot $(b b+b b+b b)$ \\
\hline Area, $b b_{1}$ & $10^{19} \mathrm{~cm}^{2}$ & $3.4 \pm 0.3$ & $4.5 \pm 0.3$ \\
Area, $b b_{2}$ & $10^{16} \mathrm{~cm}^{2}$ & $1.0 \pm 0.1$ & $1.7 \pm 0.2$ \\
Area, $b b_{3}$ & $10^{13} \mathrm{~cm}^{2}$ & - & $1.7 \pm 0.5$ \\
$k T, b b_{1}$ & $\mathrm{keV}$ & $0.120 \pm 0.001$ & $0.116 \pm 0.001$ \\
$k T, b b_{2}$ & $\mathrm{keV}$ & $0.382 \pm 0.003$ & $0.342 \pm 0.005$ \\
$k T, b b_{3}$ & $\mathrm{keV}$ & - & $1.05 \pm 0.07$ \\
$L_{\mathrm{X}}$, tot & $10^{39} \mathrm{erg} \mathrm{s}^{-1}$ & $5.3 \pm 0.5$ & $6.2 \pm 0.5$ \\
$N_{\mathrm{H}}$ & $10^{21} \mathrm{~cm}^{-2}$ & $3.64 \pm 0.05$ & $3.84 \pm 0.05$ \\
$C$-stat/d.o.f. & & $4671 / 1136$ & $4488 / 1132$
\end{tabular}

Note. $L_{\mathrm{X}(0.3-10 \mathrm{keV})}$ luminosities are calculated assuming a distance of $3.3 \mathrm{Mpc}$ and are corrected for absorption (or de-absorbed). The best-fitting three-blackbody model and the spectra are shown in Fig. 6. Both models are shown in the SED modelling in Fig. A3.

\subsection{Time-averaged continuum: spectral modelling}

The RGS spectra of the individual observations do not provide statistics sufficient to detect and resolve the spectral residuals with high significance. The $750 \mathrm{ks}$ RGS $1+2$ stacked spectrum instead has a much higher quality and enables line detection, despite the low source flux.

We simultaneously fitted the time-averaged stacked EPIC MOS 1,2 and pn, and the RGS spectra using the absorbed double-blackbody continuum model $(h o t(b b+b b))$ adopted for the spectra of the individual observations. The hard tail above $3 \mathrm{keV}$ is more evident in the stacked data and, therefore, we accounted for it using a third, hotter $(k T \sim 1 \mathrm{keV})$, blackbody as to mimic additional hard $\mathrm{X}$ ray photons down-scattered through the disc photosphere (and/or the wind). The three-blackbody model brings the C-stat from 4671 down to 4488 for four additional degrees of freedom. In all fits, the parameters of the blackbody and the ISM absorber were coupled among the EPIC and RGS models. We left the overall normalizations of the MOS 1,2 and RGS models free to vary with respect to pn in order to account for the typical 5-10 per cent cross-calibration uncertainties among their effective areas. Details on the spectral fits for both models are reported in Table 2 and Fig. 5. In order to avoid a crowded plot, we did not include the more noisy RGS spectrum in this plot, while it is shown later in Figs 6 and 7 where the EPIC data below $1.77 \mathrm{keV}$ was ignored.

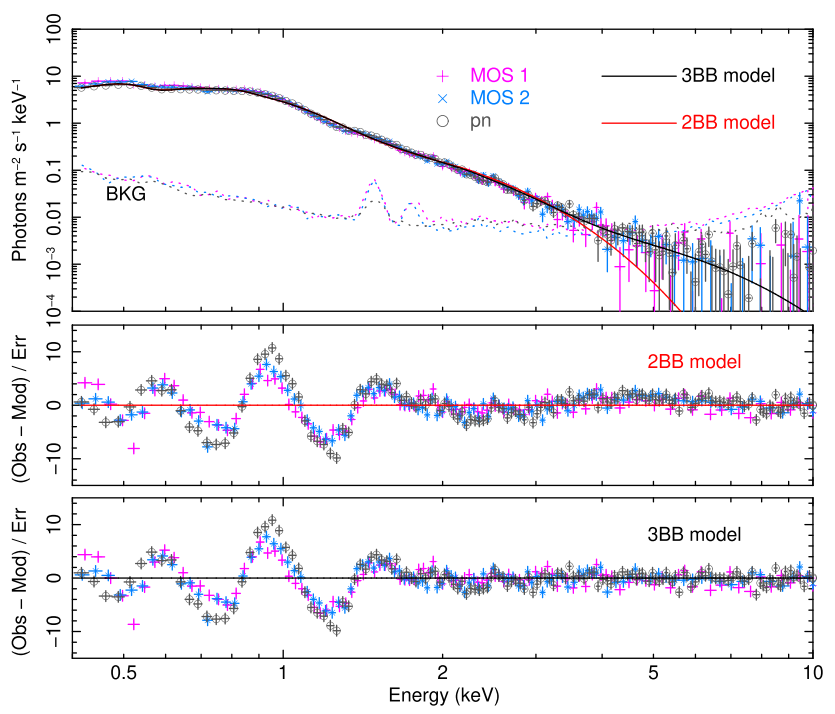

Figure 5. Time-averaged $X M M$-Newton EPIC-pn and MOS 1,2 spectra. Overlaid are two alternative continuum models consisting of two (red line) and three (black line) blackbody components.

The blackbody models used so far are the simplest available and allowed us to constrain the parameters. We tested various combination of two-component models to possibly improve the description of the spectral continuum before accounting for narrow features. These consisted of the cool blackbody component plus either a disc blackbody $(d b b)$ or a disc blackbody modified by coherent Compton scattering $(\mathrm{mbb})$ or Comptonization (comt). These did not provide improvements with respect to the three blackbody model. Similar results were provided by more complex three-component continuum models which anyway overfit the data and lead to degeneracy among model parameters due to the weak hard $(>1 \mathrm{keV})$ continuum. Alternatively, one could use the common blackbody plus powerlaw emission model. However, the power law would be very steep with a consequent unphysical divergence in the soft band, which would badly affect the ionization balance calculation (see, e.g. Pinto et al. 2020a). 


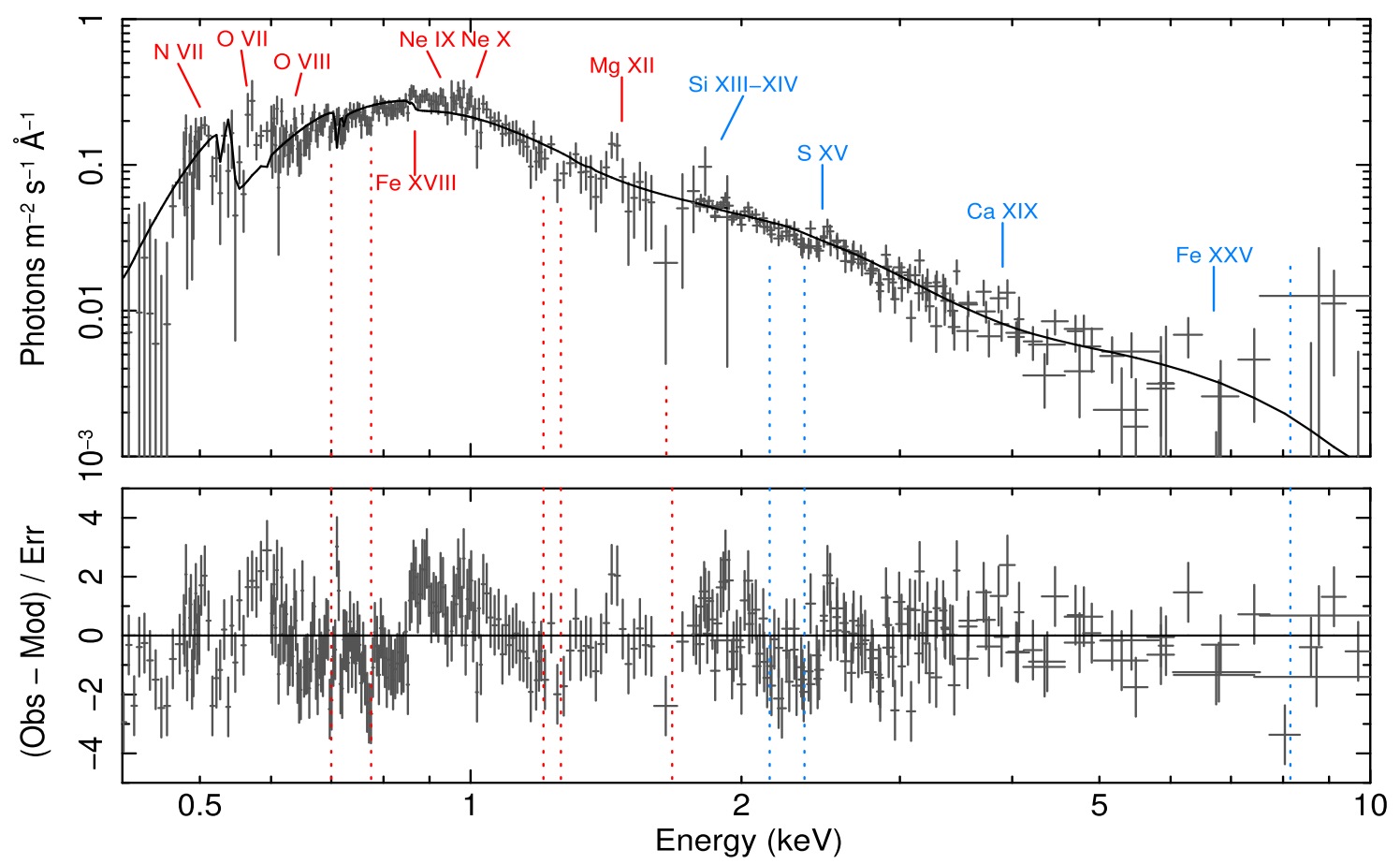

Figure 6. Top panel: time-averaged XMM-Newton RGS (0.33-2 keV) and EPIC MOS 1,2-pn (1.77-10 keV) spectra. Overlaid is the baseline three-blackbody continuum model (solid black line, see Table 2). The bottom panel shows the residuals calculated with respect to the continuum model. The rest-frame energies of the most common strong transitions in the X-ray band (red for RGS band and blue for EPIC) and the absorption features (dotted lines) are also shown. All spectra were rebinned for displaying purposes.
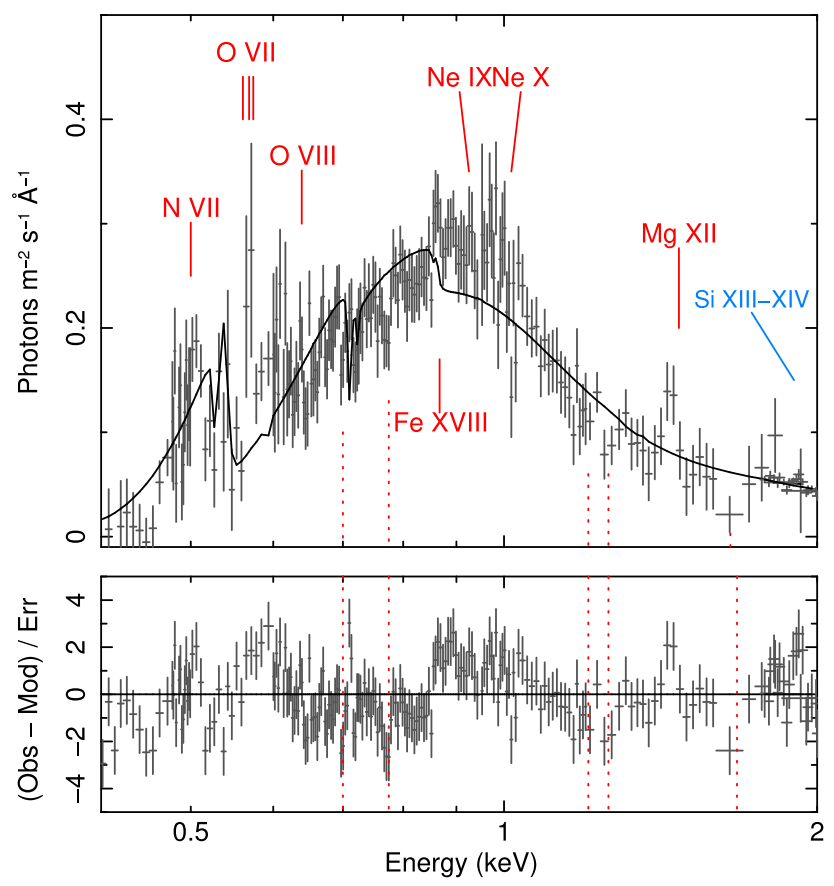

Figure 7. Time-averaged XMM-Newton RGS (0.33-2 keV) and EPIC-pn $(1.77-10 \mathrm{keV})$ spectra. Overlaid is the baseline continuum model. This is a zoom of Fig. 6 on to the RGS data.

\subsection{Time-averaged continuum: Gaussian line scan}

In Fig. 6, we show the stacked RGS and EPIC spectra, indicating the dominant $\mathrm{H}$-/He-like transitions of the $\mathrm{X}$-ray band often found in the spectra of X-ray binaries. A zoom over the RGS spectrum with adhoc linear $Y$-axis can be found in Fig. 7. The RGS exhibits substantial residuals at the same energies as the EPIC residuals but resolves them in a structure of lines, although the former have lower count rates. Strong emission-like features appear near the transition energies of the most relevant neon $\mathrm{K}$ and iron $\mathrm{L}$ lines. Additional features may be related to $\mathrm{N}$ VII and O VII-VIII, although the background starts to be important in the RGS below $0.6 \mathrm{keV}$. Some possible absorptionlike features are indicated with vertical dotted lines. The very good agreement between the positions of the RGS, MOS 1,2 and pn residuals rules out instrumental dominant features.

Following the approach used in Pinto et al. (2016), we searched for narrow spectral features by scanning the spectra with Gaussian lines. We adopted a logarithmic grid of 1000 points with energies between $0.3(41 \AA)$ and $10 \mathrm{keV}(1.24 \AA)$. This choice provided a spacing that is comparable to the RGS and EPIC resolving power in the energy range we are investigating $\left(R_{\mathrm{RGS}} \sim 100-500\right.$ and $R_{\text {EPIC }} \sim$ $20-60)$. We tested line widths $\left(\sigma_{\mathrm{G}}=\mathrm{FWHM} / 2.355\right)$ of 100,250 , 500 , and $1000 \mathrm{~km} \mathrm{~s}^{-1}$, which are comparable to the RGS resolution. At each energy we recorded the $\Delta C$ improvement to the best-fitting continuum model and expressed the significance as the square root of the $\Delta C$. This provides the maximum significance for each line (as it neglects the number of trials). We multiplied $\sqrt{\Delta C}$ by the sign of the Gaussian normalization to distinguish between emission and absorption lines.

In Fig. 8, we show the results of the line scan obtained for the time-averaged stacked RGS + EPIC spectra using the three- 


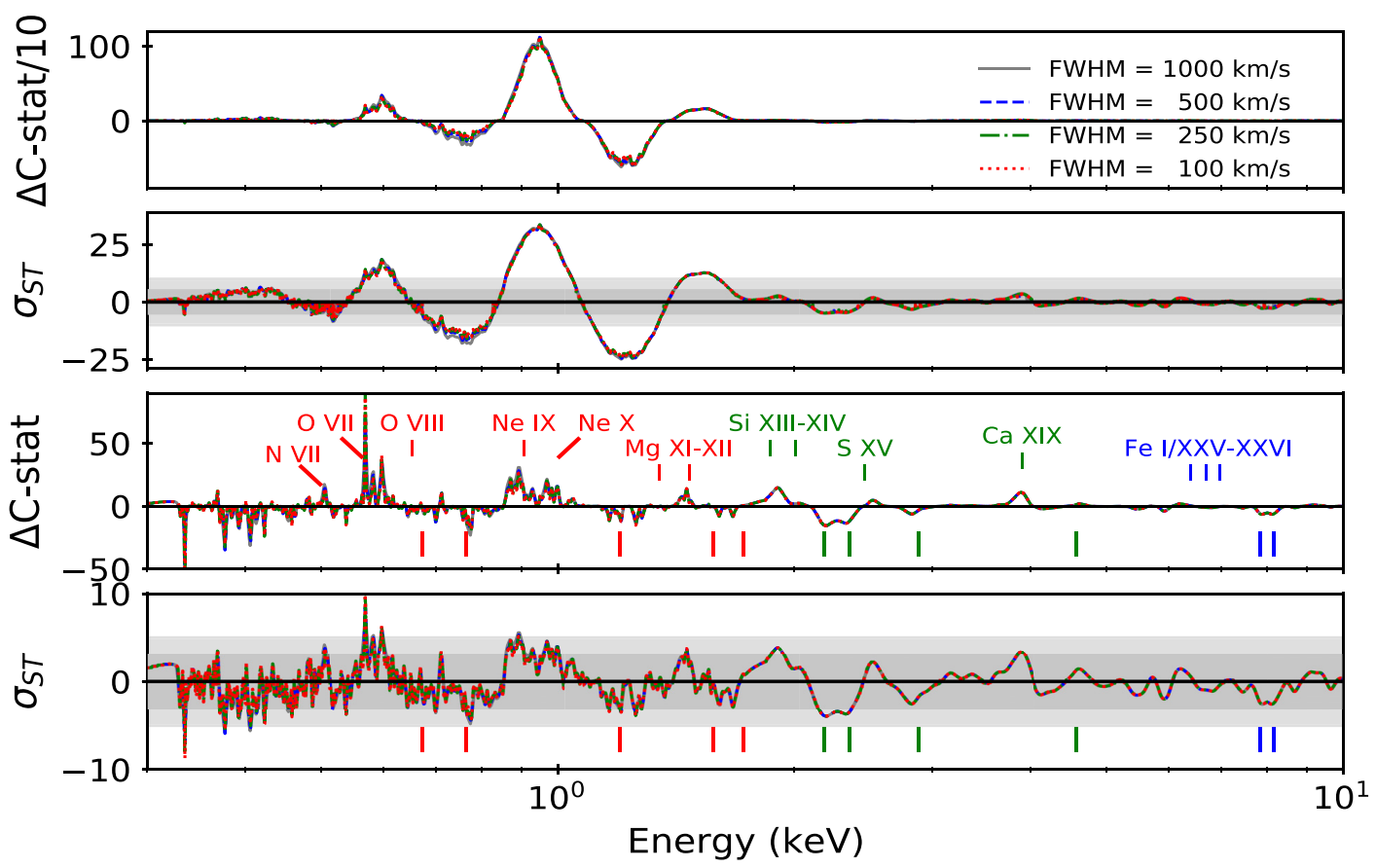

Figure 8. Gaussian line scan performed on the time-averaged XMM-Newton EPIC and RGS spectra of NGC 247 ULX-1. The top panels show the case when the EPIC spectra are used throughout the whole $0.3-10 \mathrm{keV}$ band, while the bottom two panels show the results obtained with RGS between 0.33 and $2 \mathrm{keV}$ and EPIC-pn from 1.77 to $10 \mathrm{keV}$ (see also Fig. 6). The results for four different line widths are shown. No remarkable difference is observed among the adopted widths. The single-trial line significance (' $\sigma_{\mathrm{ST}}$ ') is calculated as square root of the $\Delta C$ times the sign of the Gaussian normalization (positive/negative for emission/absorption lines). Labels are red for RGS, green for strong EPIC features and blue for the faint Fe K. The grey shaded areas show the 3 and $5 \sigma$ ST limits for individual lines.

blackbody continuum model. We performed the line scan in two ways: the first run using all RGS data $(0.3-2 \mathrm{keV})$ and EPIC data $(0.3-10 \mathrm{keV})$ and the second one ignoring the EPIC data between 0.33 and $1.77 \mathrm{keV}$, where the RGS effective area is well calibrated. When fitting only RGS in the $0.33-1.77 \mathrm{keV}$ energy band we always fixed the temperatures of the blackbody components to the bestfitting values obtained using the EPIC data in the whole $0.3-10 \mathrm{keV}$. This is due to the low count rate of the RGS spectra that limited our capability to constrain the overall continuum level and shape (see Pinto et al. 2020b).

The line scan of the EPIC + RGS spectra data picked out the strong emission-like features near $1 \mathrm{keV}$ and other two around 0.6 and $1.5 \mathrm{keV}$. Broad absorption features were also found around 0.7 and $1.2-1.3 \mathrm{keV}$ as previously done in the spectrum of each observation (see Fig. 4 and Table A1).

Owing to the low spectral resolution and high count rate of EPIC, the features appear very broad $(\sim 0.1 \mathrm{keV})$ in the EPIC + RGS Gaussian scan preventing us from identifying them. This becomes easier above $2 \mathrm{keV}$ due to the increasingly higher EPIC spectral resolution. The Gaussian scan performed using only RGS between 0.33 and $1.77 \mathrm{keV}$ resolved the features into a forest of lines. Multiple lines are responsible for the $1 \mathrm{keV}$ emission-like and the 1.2-1.3 keV absorption-like features. Interestingly, most absorption features are consistent with some Lyman $\alpha$ transitions also seen in emission, if we assume a systemic blueshifted absorption of about $0.17 c$ (see vertical ticks in Fig. 8). The $0.6 \mathrm{keV}$ features might either be interpreted as a blueshifted $\mathrm{O}$ VII $\alpha$ triplet or redshifted $\mathrm{O}$ VII $\beta+\mathrm{O}$ VIII Ly $\alpha$ emission lines. The emission lines found between $0.9-1 \mathrm{keV}$ are most likely from Ne IX-X and Fe XVIII-XXIV ions. Alternative interpretations correspond to different velocities of the X-ray emitting (and absorbing) plasmas. The use of physical models is necessary to distinguish among the several interpretations. The most simple physical models involve the adoption of plasma in either collisionally ionization equilibrium (CIE) or photoionization equilibrium (PIE).

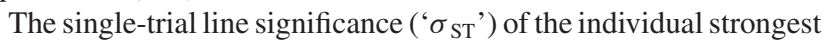
features is around $5 \sigma$, which of course is smaller if we take into account the look-elsewhere effect. However, plasma models are able to model multiple lines, combining their individual $\Delta C$ improvements to the best-fitting continuum, and boost the overall significance (see below).

\subsection{Collisional-ionization jet modelling}

Pinto et al. (2020b) performed automated scan models using either CIE or PIE plasmas. This technique prevents the fits from getting stuck in local minima, although is computationally expensive (lasting a few hours on one CPU).

\subsubsection{Collisionally ionized emitting gas}

Following Kosec et al. (2018b) and Pinto et al. (2020b), we performed a multidimensional automated scan with an emission model that assumes collisional ionization equilibrium (cie model in SPEX). We adopted a logarithmic grid of temperatures between 0.1 and $5 \mathrm{keV}$ (50 points), and line-of-sight velocities, $v_{\mathrm{LOS}}$, between $-0.3 c$ (blueshifted jet) and $+0.3 c$ (redshifted jet, with steps of $500 \mathrm{~km} \mathrm{~s}^{-1}$ ). We tested several values of velocity dispersion (from 100 to $10000 \mathrm{~km} \mathrm{~s}^{-1}$ ), finding comparable results as already shown by the Gaussian line scan in Fig. 8, with the best fit achieved at $v_{\mathrm{RMS}} \sim 3000 \mathrm{~km} \mathrm{~s}^{-1}$. Abundances were chosen to be Solar (to limit 

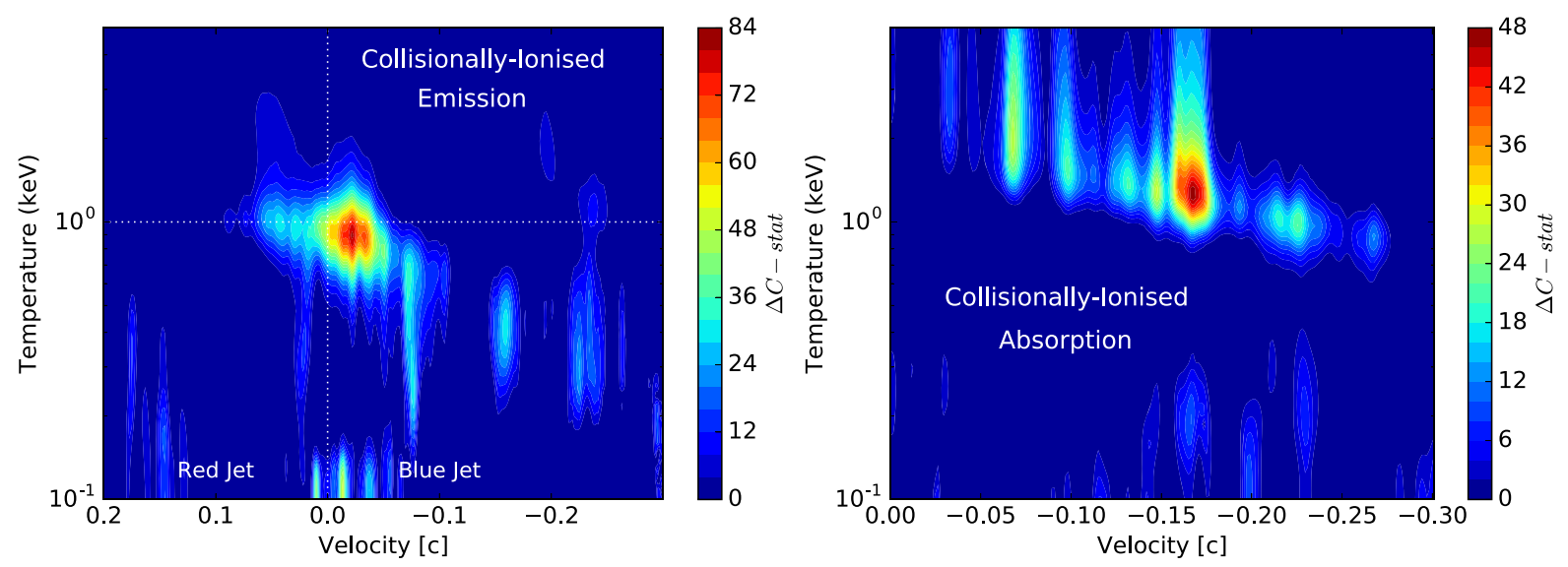

Figure 9. Multidimensional scans of collisional-ionization emission (left-hand panel) and absorption models (right-hand panel) for NGC 247 ULX-1 timeaveraged EPIC + RGS spectra. The X-axis shows the line-of-sight velocity (negative means blueshift, i.e. motion towards the observer). The colour is coded according to the $\Delta C$ fit improvement to the spectral continuum model.

the computing time) and the emission measure $E M=n_{\mathrm{e}} n_{\mathrm{H}} V$ was the only free parameter of the CIE in the spectral fit.

We applied the automated routine scanning CIE models on to the NGC 247 ULX-1 time-averaged RGS (0.33-2 keV) and EPIC MOS 1,2-pn (1.77-10 keV) spectra. We adopted the three-blackbody continuum model shown in Section 3.2 and Table 2 (see also black line in Fig. 6). The results are shown in Fig. 9 (left-hand panel). The best-fitting corresponds to a large improvement with respect to the continuum model $(\Delta C=82$, for four additional degrees of freedom) and was achieved for a CIE temperature of $0.9 \mathrm{keV}$ and a small blueshift of around $6500 \mathrm{~km} \mathrm{~s}^{-1}(\sim 0.022 c)$. These results were driven by the strong lines that can be seen in the RGS spectrum $\lesssim$ $1 \mathrm{keV}$ in Fig. 8 (bottom two panels).

We have checked our method by testing an identical CIE scan on the well-known Galactic X-ray source SS 433. This source is not ultraluminous in the X-ray band due to obscuration of the accretion disc from local circumstellar gas, but exhibits a persistent and bright $\left(10^{40} \mathrm{erg} \mathrm{s}^{-1}\right)$ radio jet, which is powering a luminous optical superbubble (Brinkmann, Aschenbach \& Kawai 1996; Fabrika 2004; Medvedev, Khabibullin \& Sazonov 2019). SS 433 is therefore considered to be viewed edge-on. Were it observed face-on, it would likely appear as a ULX (Begelman, King \& Pringle 2006; Poutanen et al. 2007; Middleton et al. 2018). SS 433 also shows a relativistic jet in the form of blueshifted lines from multitemperature plasma in collisional-ionization equilibrium (Marshall, Canizares \& Schulz 2002). The results obtained for SS 433 are very similar to NGC 247 ULX-1 and in agreement with Marshall et al. (2013) and Medvedev et al. (2018), which supports our method (see Appendix A3).

\subsubsection{Collisionally ionized absorbing gas}

It is uncommon to adopt absorption models of gas in collisionalionization equilibrium in accreting objects as (1) it is difficult to distinguish between photoionization and collisional ionization on the sole basis of the dominant resonant absorption lines and (2) we hardly expect any jet to absorb the X-ray source continuum along our line of sight. However, we cannot exclude that shocks are produced by interaction between the ULX wind and the stellar companion or the surrounding bubble (or within the wind itself). Therefore, we also performed a model scan with the hot model in SPEX, which works just like cie but assumes absorbing gas.
In Fig. 9 (right-hand panel), we show the results using the hot model over the same $k T$ range used for the emitting gas, adopting a velocity dispersion of $500 \mathrm{~km} \mathrm{~s}^{-1}$ and line-of-sight velocities, $v_{\mathrm{LOS}}$, ranging between $-0.3 c$ and zero, i.e. only Doppler blueshifts or outflows rather than inflows. The best-fitting solution is obtained for a $-0.17 c$ blueshifted with a remarkable $\Delta C=48$ as suggested by the detection of several negative Gaussians blueshifted by similar values in Fig. 8.

\subsection{Photoionization wind modelling}

The emission and absorption lines can be produced by winds rather than by jets as expected in the case of super-Eddington accretion discs and, therefore, in ULXs. Accurate photoionization models require knowledge of the radiation field, i.e. the SED from optical to hard $\mathrm{X}$-ray energies.

\subsubsection{SED and photoionization balance}

Following Pinto et al. (2020a,b), we built the time-averaged SED of NGC 247 ULX-1 using data from the XMM-Newton campaign and archival $H S T$ observations (as taken from Feng et al. 2016). For issues regarding the non-simultaneity of $H S T$ and XMM observations, see Appendix A4. For the X-ray band $\left(0.3-10 \mathrm{keV}\right.$ or $\left.\sim 10^{16-18} \mathrm{~Hz}\right)$ we used the best-fitting three-blackbody continuum model, estimated in Section 3.2, Fig. 5 and Table 2. As shown in Section 2.1.3, the OM filters were not sensitive enough to detect the optical counterpart, but their flux upper limits in the optical and UV energy bands are however comparable to the HST measurements (see Fig. A3 top panel). We therefore modelled the optical/UV portion of the SED with the two-blackbody model of Feng et al. (2016), which together with the three-blackbody X-ray model formed our five-blackbody SED model.

We can describe the photoionization equilibrium with the ionization parameter, $\xi$, defined as $\xi=L_{\text {ion }} /\left(n_{\mathrm{H}} R^{2}\right)$ (see, e.g. Tarter, Tucker \& Salpeter 1969), where $L_{\text {ion }}$ is the ionizing luminosity (measured between $13.6 \mathrm{eV}$ and $13.6 \mathrm{keV}$ ), $n_{\mathrm{H}}$ the hydrogen volume density and $R$ the distance from the ionizing source. The ionization balance was calculating with the SPExpion model, which calculates the transmission and the emission of a thin slab of photoionized gas, self-consistently. 

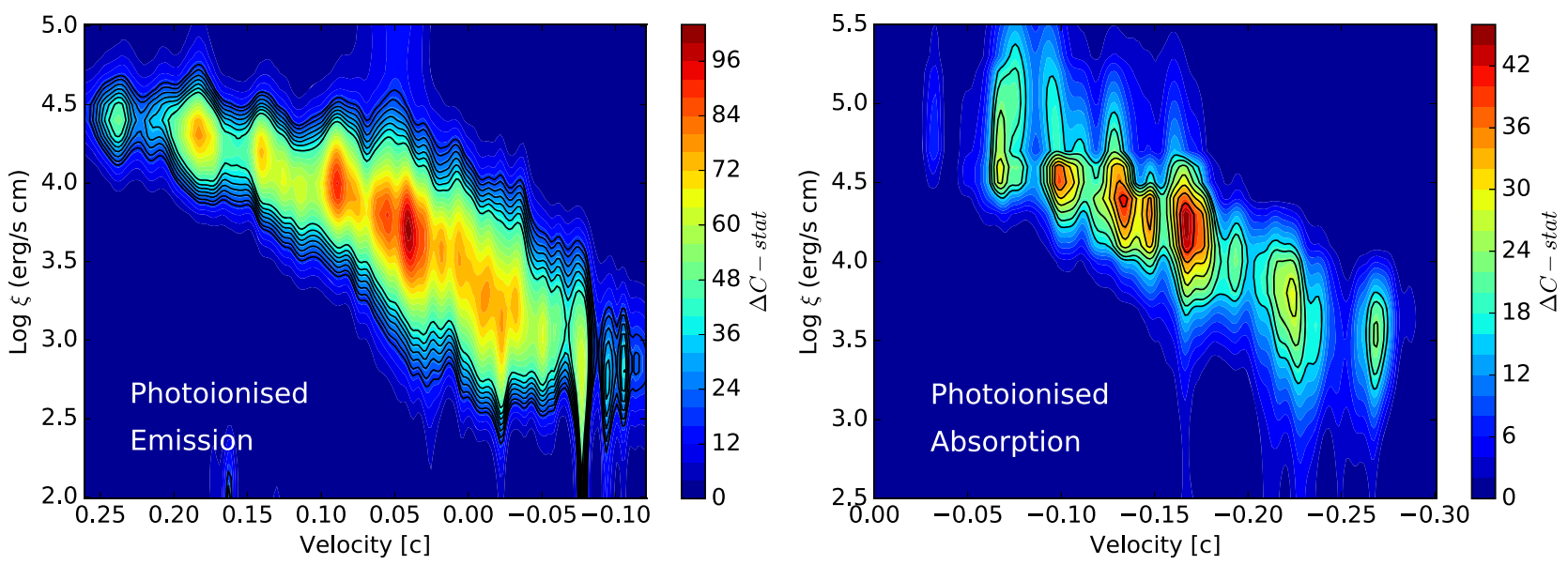

Figure 10. Scans of photoionization emission (left) and absorption (right) models for the time-averaged EPIC and RGS spectra. Labels are the same as in Fig. 9. The black contours refer to the $(2.0,2.5, \ldots 4.5,5.0) \sigma$ confidence levels estimated by Monte Carlo simulations.

Following Pinto et al. (2020b), we also computed the stability (or $S$ ) curve, which is the relationship between the temperature (or the ionization parameter) and the ratio between the radiation and the thermal pressure, which can be expressed as $\Xi=F /\left(n_{\mathrm{H}} c k T\right)=$ $19222 \xi / T$ (Krolik, McKee \& Tarter 1981). The stability curve is shown in Fig. A3 (bottom panel). The branches of the $S$ curve with a negative gradient are characterized by thermally unstable gas. In this work, we assumed that the wind is seeing the same SED that we observe and, therefore, adopted the five-blackbody model SED and ionization balance. Systematic effects from the SED choice are discussed in Section 4 and Appendix A4.

\subsubsection{Photoionized emitting gas}

Once the SED and the ionization balance were computed, we scanned the time-average EPIC + RGS spectra with the SPEX pion model with the same multidimensional routine used for the cie model in Section 3.4.1, and a similar parameter space. We adopted a logarithmic grid of ionization parameters $\left(\log \xi\left[\mathrm{erg} \mathrm{s}^{-1} \mathrm{~cm}\right.\right.$ ] between 0 and 6 with 0.1 steps). The only free parameter for the pion is the column density, $N_{\mathrm{H}}$.

Unlike NGC 1313 ULX-1, the RGS spectrum of NGC 247 ULX-1 does not exhibit well-resolved emission-line triplets. This is perhaps due to the longer integration time required and the variability of the line centroid (see Fig. 4), which could wash out the triplets when stacking all the spectra. Additionally, the crucial O VII complex is affected by the background noise. The lack of He-like triplets means that the volume density and the luminosity of the photoionized gas are degenerate. Fitting both parameters results in poor constraints and much higher computation time. We therefore chose not to fit the volume density and adopted $n_{\mathrm{H}}=10^{10} \mathrm{~cm}^{-3}$, which is a lower limit found for NGC 1313 ULX-1 (Pinto et al. 2020b). This would only slightly affect the overall flux and column density of the pion component.

The pion covering fraction is set to zero (i.e. pion only produces emission lines) and the solid angle $\Omega=4 \pi$. Fitting additional parameters such as $\Omega$ might provide even better fits but would significantly increase the computing time, without altering the velocity and ionization parameters.

In Fig. 10 (left-hand panel), we show the results obtained using a pion line width of $1000 \mathrm{~km} \mathrm{~s}^{-1}$. The confidence level (CL) is expressed in $\sigma$, which is constrained using Monte Carlo simulations (see Appendix A5). A peak $(\Delta C=77)$ corresponding to a solution of blueshift emission is seen around $0.02-0.03 c$ in agreement with the CIE model scans (see Section 3.4 and Fig. 9). However, the different ionization balance and types of emission lines in the photoionization equilibrium detected another, stronger $(\Delta C=102)$, peak corresponding to a redshift of $\sim+0.05 c$.

\subsubsection{Photoionized absorbing gas}

In principle, we could just use pion for both emission and absorption. However, this model re-calculates the ionization balance at every iteration and therefore is computationally expensive. Therefore, for the absorbing gas we chose to use the faster xabs model, which is optimized for absorption and adopts the pre-calculated ionization balance (see Section 3.5.1).

The xabs model shares several parameters with pion except the opening angle of the line emission which is zero since no emission is present in this model. We adopted a covering fraction equal to unity in order to avoid degeneracy and reduce the computing time. We calculated the grid of photoionized xabs models in the same way as the pion models, but assuming line-of-sight velocities, $v_{\text {LOS }}$, ranging between $-0.3 c$ and zero, i.e. only Doppler blueshifts as for the CIE hot absorption models in Section 3.4.2.

In Fig. 10 (right-hand panel), we show the probability distributions from scans of the RGS and EPIC spectra with $v_{\text {RMS }}=1000 \mathrm{~km} \mathrm{~s}^{-1}$. As expected, our code confirmed the $v=-0.17 c$ solution $(\Delta C=$ 46).

\subsection{Final fits with physical plasma models}

In order to check the inter-dependence of the emitting and absorbing plasma components and to test for any variations in the values of their parameters we performed two more fits of the RGS and EPIC data (with EPIC still excluded between $0.33-1.77 \mathrm{keV}$ ) adding on to the 3-blackbody continuum two alternative plasma models. The first one was a wind model that used the pion in emission and the $x a b s$ in absorption. The second model was an approximation of jets and shocks in the form of cie component in emission and hot component in absorption. The results for the two models are shown in Table 3 and Fig. 11 (zoomed on to the RGS). The absorption components 
Table 3. NGC 247 ULX-1: alternative plasma models.

\begin{tabular}{lccc}
\hline Model 1 & Parameter & Emission & Absorption \\
\hline PIE & $L_{\mathrm{X}}(E), N_{\mathrm{H}}(A)$ & $1.4 \pm 0.2$ & $2.8 \pm 0.1$ \\
(wind) & $\log \xi\left(\mathrm{erg} \mathrm{s}^{-1} \mathrm{~cm}\right)$ & $3.7 \pm 0.1$ & $4.3 \pm 0.1$ \\
& $v_{\mathrm{LOS}}(c)$ & $+0.042 \pm 0.004$ & $-0.166 \pm 0.002$ \\
& $v_{\mathrm{RMS}}\left(\mathrm{km} \mathrm{s}^{-1}\right)$ & $3000_{-800}^{+2700}$ & $400 \pm 200$ \\
& $\Delta C^{a}\left({ }^{e}\right)$ & $104(97)$ & $46(32)$ \\
& $\sigma^{a}\left({ }^{e}\right)$ & $>5(>5)$ & $>5(>4)$ \\
Model 2 & Parameter & Emission & Absorption \\
CIE & $L_{\mathrm{X}}(E), N_{\mathrm{H}}(A)$ & $1.4 \pm 0.2$ & $2.5 \pm 0.1$ \\
(jet) & $k T(\mathrm{keV})$ & $0.9 \pm 0.1$ & $1.3 \pm 0.1$ \\
& $v_{\mathrm{LOS}}(c)$ & $-0.022 \pm 0.002$ & $-0.168 \pm 0.003$ \\
& $v_{\mathrm{RMS}}\left(\mathrm{km} \mathrm{s}^{-1}\right)$ & $3000_{-1000}^{+3000}$ & $1600 \pm 850$ \\
& $\Delta C^{a}\left(e^{e}\right)$ & $83(51)$ & $48(32)$ \\
& $\sigma^{a}\left(e^{e}\right)$ & $>5(>5)$ & $>5(>4)$
\end{tabular}

Notes. Main properties of the plasma components for two alternative models. Model 1 or PIE/wind: photoionized emission (pion) and absorption (xabs, see Figs 10 and 11). Model 2 or CIE/jet: collisionally ionized emission (cie) and absorption (hot, see Fig. 9). The column densities, $N_{\mathrm{H}}$, are in $10^{22} \mathrm{~cm}^{-2}$. The line-of-sight velocities, $v_{\mathrm{LOS}}$, are in units of light speed $c$; the velocity dispersion, $v_{\mathrm{RMS}}$, is in $\mathrm{km} \mathrm{s}^{-1}$. The $0.3-10 \mathrm{keV}$ luminosities of the emitting plasmas, $L_{X}(E)$, are defined in units of $10^{38} \mathrm{erg} \mathrm{s}^{-1}$. The $\Delta C^{a}\left({ }^{e}\right)$ refer to the $\Delta C$-statistics of each component computed when the component is the only one in model (a) or when the other one is included (e). The same applies to the detection significances, $\sigma^{a}\left({ }^{e}\right)$, evaluated with Monte Carlo simulations.

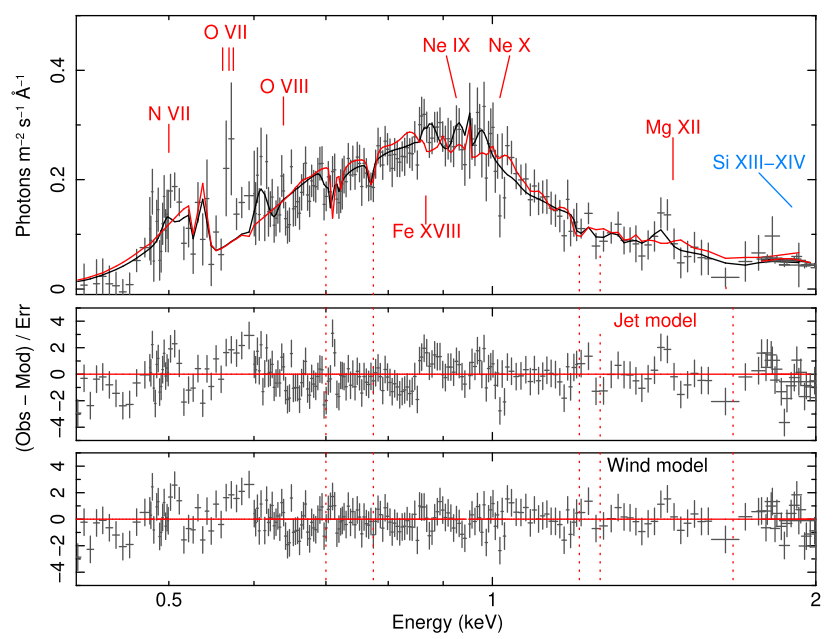

Figure 11. Time-averaged XMM-Newton RGS (0.33-2 keV) and EPIC-pn $(1.77-10 \mathrm{keV})$ spectra. Overlaid are two alternative models (jet - CIE, red line and the wind - PIE, black line). The spectra were regrouped and the plot zoomed on to the RGS data and the $0.4-2 \mathrm{keV}$ energy band for displaying purposes.

provided very similar results, especially for the column densities and the line-of-sight velocities. As previously noted, the emission components show some differences. The results are discussed in Section 4.

The temperatures of the three blackbody components were always fixed to the EPIC $0.3-10 \mathrm{keV}$ fits (Table 2) each time we ignored EPIC data between $0.33-1.77 \mathrm{keV}$, resulting in parameters consistent with the continuum modelling.

By alternatively excluding one particular plasma component from the spectral model we estimated the relative contribution to the spectral fit and the minimum $\Delta C$-stat improvement of each component for both the wind and jet model (see $\Delta C^{e}$ values in Table 3). Following
Table 4. NGC 247 ULX-1: continuum and plasma models.

\begin{tabular}{lcc}
\hline Model & RGS + EPIC $(>1.77 \mathrm{keV})$ & RGS + EPIC (full band) \\
\hline $3 b b$ & $2019 / 994$ & $4488 / 1132$ \\
$3 b b *$ hot & $1971 / 990$ & $3043 / 1132$ gas par fixed \\
$3 b b * h o t+c i e$ & $1895 / 886$ & $2542 / 1132$ gas par fixed \\
$3 b b * x a b s$ & $1973 / 990$ & $2233 / 1124$ \\
$3 b b * x a b s+$ pion & $1877 / 886$ & $3033 / 1132$ gas par fixed \\
& & $2431 / 1132$ gas par fixed \\
\hline
\end{tabular}

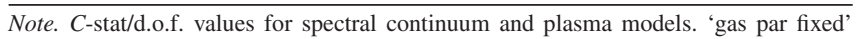
means that for those fits the parameters of both emission and absorption components were fixed to the best-fitting values obtained excluding EPIC data below $1.77 \mathrm{keV}$.

Pinto et al. (2020b), we compared the minimum $\Delta C$-stat values with the Monte Carlo simulations to obtain an approximate estimate of the minimum significance of each wind or jet component (parameter $\left.\sigma^{e}\right)$, which is highest for the emission phases.

Finally, to further test the strength of our results we performed a fit of the RGS and EPIC data including the whole EPIC energy band but fixing the plasma models to the best-fitting results of the hybrid RGS (0.33-2 keV) plus EPIC (1.77-10 keV) fits. In Section 3.5.3, we noted that the inclusion of the photoionized xabs absorption component significantly decreased the C-stat from 4488 (of the simple three-blackbody model) to 3033. The addition of the photoionized pion emission component further lowered the $\mathrm{C}$-stat to 2431 (with a $\chi^{2}=1830$ and a total of 1132 degrees of freedom). We performed the same fit by using instead the RGS jet model with the hot collisionally ionized component fixing the parameters to those in Table 3. This decreased the C-stat from 4488 to 3043, similarly to the photoionized absorber. The addition of a cie emission component (with fixed plasma parameters) implied a final C-stat $=2542$, which is slightly worse than pion due to some positive residuals left around $0.9 \mathrm{keV}$ that can also be seen in the RGS spectral modelling in Fig. 11.

We notice that all the spectral fits shown here are not formally acceptable (see Table 4), although the winds components provide significant improvements. One reason is the variability of the features, both of their centroids and relative strength (see Figs 3 and 4). This means that more complex models would be required to correctly fit the lines. On the other hand, the winds are likely multiphase as shown by the low-temperature O VII clearly missed by our single phase model (see Fig. 11). This was already shown in Pinto et al. (2020b) and occurs in SS 433 too (see Appendix A3). Finally, some bad cross-calibration below $0.6 \mathrm{keV}$ between the EPIC and RGS cameras further prevent us from obtaining fully acceptable fits (see Fig. 5).

\section{DISCUSSION}

It is still unclear how does the wind vary with the accretion rate and whether it has a major role in shaping ULX spectra. Pinto et al. (2020a), Pinto et al. (2020b) showed that the wind evolves with the changes in the continuum from the fainter, harder states to the brighter states, which implies a tight relationship between the source's spectral continuum and wind appearance as observed by comparing winds in different ULXs.

Among the ULXs, the SSUL X-ray sources are particularly fascinating objects. The fact that such sources reach very high luminosities (several $10^{39} \mathrm{erg} \mathrm{s}^{-1}$ ) but always exhibiting very soft $(k T \sim 0.1 \mathrm{keV})$ spectra indicates that they are being observed at moderate-to-high inclination angles as also suggested by the presence of dips in their light curves (see, e.g. Feng et al. 2016). In fact, 
Urquhart \& Soria (2016) modelled the soft X-ray residuals and the $\sim 1 \mathrm{keV}$ drop found in several CCD spectra of ULSs with a model of thermal emission and an absorption edge, which they interpreted as a result of absorption and photon reprocessing by an optically thick wind which obscures the innermost regions where most hard X-rays are produced.

\subsection{Time evolution of the $1 \mathrm{keV}$ residuals}

The stacked XMM-Newton light curve (see Fig. 2) shows different pattern of source variability such as a long-term overall change in the average flux on daily time-scales followed by abrupt drops in the flux where the source becomes softer (the dips) on time-scales between $100 \mathrm{~s}$ and a few hours. The dipping activity seems also to enhance during observations with higher flux peaks. The higher flux might be associated with a higher local accretion rate, which then would increase the radiative force and launch optically thick wind cloudlets in the line of sight, thereby obscuring the innermost regions of the disc responsible for the hard X-ray emission (as suggested by Urquhart \& Soria 2016). More insights on the nature of the dips will be provided by Alston et al. (2021). This work shows that the dips in the higher flux observations preferentially occur on 5 and $10 \mathrm{ks}$ time-scales, which suggests that they are caused from obscuration at $\sim 10^{4-5} R_{\mathrm{G}}$, where $R_{\mathrm{G}}$ is the Gravitational radius (if the time-scales are associated with Keplerian motion around an NS or a stellar-mass $\mathrm{BH})$. Such a range is comparable to the distance that the $0.17 c$ wind would travel on a time-scale of $1 \mathrm{ks}$, suggesting a possible connection between them.

The high-quality EPIC spectra of the individual observations show a remarkable flux variability in the features around $1 \mathrm{keV}$ (see Fig. 3). In order to quantify such variability, we modelled the two strongest absorption features around 0.7 and $1.2 \mathrm{keV}$, and the dominant emission-like feature at $1 \mathrm{keV}$ with three Gaussian lines for the EPIC (MOS and pn) spectral of the individual observations. All lines show a distinct pattern with their energy centroids significantly blueshifted during the brightest observations (which also exhibit most dips and the highest variability, see Fig. 4). Interestingly, the fluxes of the high-energy lines ( 1 and $1.2 \mathrm{keV}$ ) significantly decrease during the dipping observations while the $0.7 \mathrm{keV}$ line seems to strengthen (see Table A1). This would either suggest a different location of the three lines, with the $0.7 \mathrm{keV}$ line coming from the outer and less obscured regions, or a change in the ionization state of the absorber during the high-flux periods. This is similar to what was observed in NGC 1313 ULX-1 (Middleton et al. 2015b; Pinto et al. 2020b). A detailed study of the broad-band spectra and residuals evolution will be shown by D'Aì et al. (in preparation). The fact that the location and strength of the residuals vary on hourly time-scales with the source flux provides strong evidence in support for a disc wind rather than emission from the local ULX bubble or the galactic ISM.

\subsection{A wind or a jet?}

\subsubsection{Emission lines}

The time-average stacked RGS spectrum showed strong emission residuals near the transition energies of several ions such as O VII-VIII, Ne IX-X, and Fe XVIII (see Figs 7 and 8). The agreement between RGS and EPIC is corroborated by applying the wind model constrained using only RGS in the $0.33-1.77 \mathrm{keV}$ band to the whole EPIC MOS and pn time-average spectra (see Table 4). Unfortunately, the He-like emission triples of e.g. O VII and Ne IX are not well resolved likely due to the stacking of RGS spectra from different observations that clearly showed some variability in the line centroid as discussed above. This limited our capabilities of distinguishing between collisional and photoionization, but the use of full plasma models provided some constraints.

By performing automated searches of plasma models in a large parameter space, we built probability contours for both collisionally ionized and photoionized plasma emission models. The properties of the line-emitting gas are very similar to those of the Galactic superEddington source SS 433 with a low velocity along the line of sight and a mild $1 \mathrm{keV}$ temperature which is expected by the strong $\mathrm{Ne} \mathrm{K}$ and $\mathrm{Fe} \mathrm{L} \mathrm{emission} \mathrm{around} 1 \mathrm{keV}$ (see Fig. 9). It is well established that the emission lines of SS 433 are from the jet with the low velocity indicating that the precessing jet was at very high angle, close to $90^{\circ}$, in the analysed observation. If the lines of NGC 247 ULX-1 were also from a variable jet, the observed low velocity would suggest that it is being viewed at high angle in agreement with the presence of dips.

The photoionization emission models (pion component in SPEX, see Fig. 10) however provided a significantly higher improvement to the spectral fits and a better description of the emission lines (see Table 3 and Fig. 11). This together with the evolution of the lines with the source continuum would favour photoionization equilibrium similarly to the emission lines in NGC 1313 ULX-1 (Pinto et al. 2020b).

Regardless of the adopted equilibrium state, the luminosity of the line-emission component is remarkably high $\left(L_{0.3-10 \mathrm{keV}}>\right.$ $10^{38} \mathrm{erg} \mathrm{s}^{-1}$ ), similarly to NGC 1313 ULX-1, NGC 5408 ULX-1 (Pinto et al. 2016), NGC 55 ULX-1 (Pinto et al. 2017), NGC 5204 ULX-1 (Kosec et al. 2018a), and other ULXs (e.g. Wang, Soria \& Wang 2019). This is about two to three orders of magnitude higher than the emission lines in SS 433 and those producing the winds in classical supergiant X-ray binaries (sub-Eddington neutron stars accreting from supergiant OB stars, e.g. El Mellah \& Casse 2017) or the lines from accretion disc coronae of low-mass X-ray binaries (see, e.g. Psaradaki et al. 2018). The luminosity of $1.4 \times 10^{38} \mathrm{erg} \mathrm{s}^{-1}$ is instead comparable to the extended $\mathrm{X}$-ray emission recently found around the extremely bright pulsating NGC 5907 ULX-1 (Belfiore et al. 2020), which suggests that the wind might be energetic enough to mechanically drive the $\sim 100$-pc super bubbles (see also Pinto et al. 2020a).

Similarly to NGC 1313 ULX-1, the O VII emission lines cannot be reproduced with the emission component responsible for the $\mathrm{Fe} \mathrm{L}$ and Ne K emission (see Fig. 11). A second component (either photoor collisionally ionized) with a low blueshift of $6000 \mathrm{~km} \mathrm{~s}^{-1}$ would be required.

\subsubsection{Absorption lines}

In this work we also reported a highly significant detection of mildly relativistic, ultrafast, outflows. Both collisional and photoionization (see Figs 9-11) plasma models identified a high-velocity outflow $(-0.17 c)$ in the range of the velocities found in other ULX winds.

The ionization parameter is rather high $(\log \xi=4.3)$ which is not surprising given the soft SED adopted for this source (see Fig. A3). If the wind at the launch is seeing a different SED (e.g. the hot innermost regions presumably obscured along our line of sight) the overall ionization balance might be significantly different. This subject was extensively discussed in Pinto et al. (2020a) who found larger instability branches in the $S$ curves of harder ULXs. Therefore, as a test, we performed an additional fit with the photoionized pion $+x a b s$ wind model (as previously done in Section 3.6) by adopting 

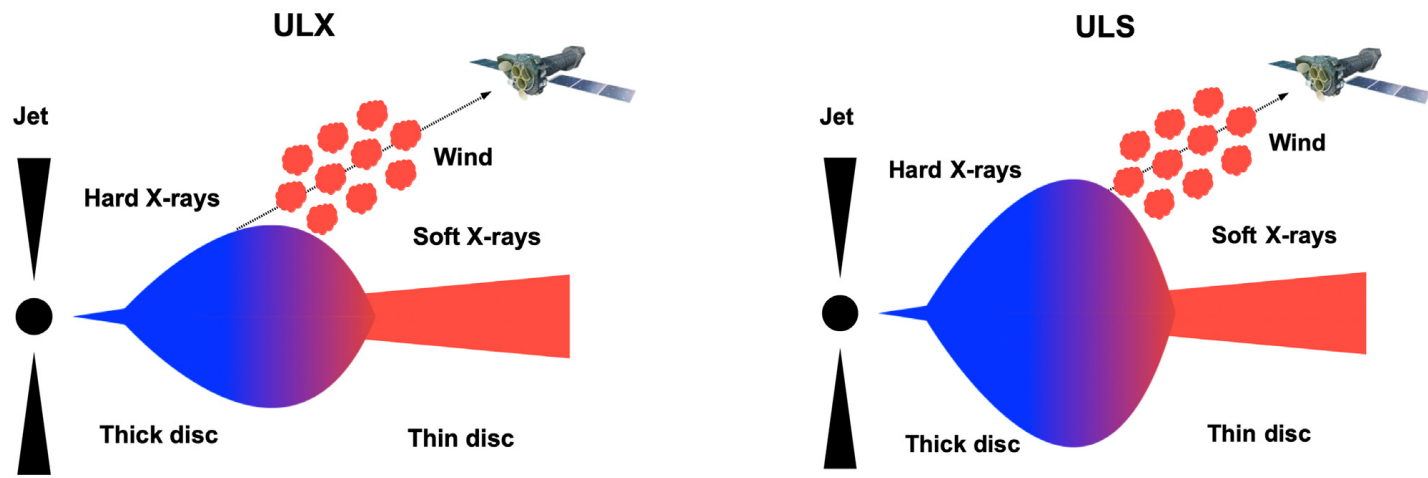

Figure 12. A possible scenario for the dips and ULX-ULS transitions in NGC 247 ULX-1. The source is observed at a viewing angle that is high enough that the inner disc is already partly obscured by the wind (soft ULXs, left-hand panel). An increase of the accretion rate pushes up the scale height of the disc and the optically thick base of the wind, causing an near-total obscuration of the inner regions and the source appears as an ultraluminous supersoft source (ULS, right-hand panel; see also Pinto et al. 2017, 2020b; Guo et al. 2019).

the ionization balance calculated for the hard state of NGC 1313 ULX-1 in Pinto et al. (2020b) to estimate the systematic effects on the wind parameters. The fit was statistically indistinguishable from the one performed with the ionization balance computed for NGC 247 ULX-1, with the exception of the ionization parameters which, as expected, turned out to be significantly lower by about $\Delta \log \xi \sim 1$.

The absorption lines are generally weaker than the emission lines in the RGS spectrum of NGC 247 ULX-1 which could be due to the low source continuum (Kosec et al. submitted). This was also predicted by Pinto et al. (2017) as the lines are normally seen on top of the continuum from the innermost regions which in this case is likely obscured.

Statistically we cannot distinguish photoionization from collisional ionization, but the former is favoured by the photoionized nature of the emitting plasma and the unusual detection of collisionally ionized absorption in XRB winds.

\subsection{Accretion disc and wind physics}

In the framework of super-Eddington accretion the luminosity scales with the logarithm of the accretion rate in Eddington units $(\dot{m}=$ $\dot{M} / \dot{M}_{\mathrm{E}}$ ) times the geometrical beaming of the funnel created by the height of the disc around the spherization radius and by the wind itself (see Fig. 12). Following King \& Lasota (2020), the apparent luminosity can be expressed with $L_{\text {app }}=L / b=L_{\mathrm{E}}(1+\ln \dot{m}) / b$, where $L$ is the intrinsic luminosity, $L_{\mathrm{E}}$ the luminosity in Eddington units, and $b=73 / \dot{m}^{2}$ the geometrical beaming.

To estimate the bolometric luminosity of NGC 247 ULX-1 we integrated the broad-band SED between $1 \mathrm{eV}$ and $10 \mathrm{keV}$ (or $2.4 \times 10^{14-18} \mathrm{~Hz}$, see Fig. A3) and obtained $9.4 \times 10^{39} \mathrm{erg} \mathrm{s}^{-1}$. NGC 247 ULX-1 luminosity could therefore be explained by assuming a black hole accreting above 10 times the Eddington rate or a neutron star accreting above $\dot{m}=25$. At $\dot{m} \sim 10$ the spherization radius, i.e. the base of the wind, would be $R_{\mathrm{sph}}=27 / 4 \dot{m} R_{\mathrm{G}}=68 R_{\mathrm{G}}$. Interestingly, this is very close to the escape radius for a $-0.17 c$ wind $\left(R_{\mathrm{e}}=2 G M / v^{2}=2 R_{\mathrm{G}} c^{2} / v^{2}=73 R_{\mathrm{G}}\right)$, which would indeed suggest that we detected a wind launched from the spherization radius of a compact object above $10 \dot{M}_{\mathrm{E}}$.

From equation (38) in Poutanen et al. (2007), assuming $M_{\mathrm{BH}}=$ $10 M_{\odot}$ and $\dot{m}=10$, we estimated a temperature for the spherization radius $T_{\text {sph }} \sim 0.3 \mathrm{keV}$, which is comparable to the warm blackbody component in our fits (see Table 2$)$, with the cooler $(\sim 0.1 \mathrm{keV})$ blackbody associated with the outer disc and, likely, the wind photosphere as suggested by recent work (see, e.g. Qiu \& Feng 2021, Gúrpide et al. 2021). We notice, however, that the source is being seen at high inclination with a substantial fraction of the hard $\mathrm{X}$-ray photons obscured by the funnel. The intrinsic luminosity of NGC 247 ULX-1 might therefore be higher than the value estimated above with a higher accretion rate, implying $T_{\text {sph }} \sim 0.1-0.2 \mathrm{keV}$, closer to the cooler blackbody component, and a slightly larger $R_{\text {sph }}$. It is also possible that the wind is launched with lower velocity at radii larger than $73 R_{\mathrm{G}}$ and it gets accelerated by radiation pressure from the inner accretion flow (see, e.g. Takeuchi et al. 2013).

Similar considerations would apply to a non-magnetar $\left(B \lesssim 10^{12} \mathrm{G}\right)$ neutron star with $\dot{m}=25$ since the spherization radius (in $\mathrm{cm}$ ) would be of the same order of magnitude as a $10 \mathrm{M}_{\odot}$ black hole as both $R_{\text {sph }}$ and $T_{\text {sph }}$ scale with the $\dot{M}$ and the mass of the compact object, whose trends nearly cancel out. This was briefly discussed in Pinto et al. (2020a).

The kinetic power of the wind can be written as $L_{w}=1 / 2 \dot{M}_{w} v_{w}^{2}=2 \pi m_{p} \mu \Omega C v_{w}^{3} / \xi L_{\text {ion }} \sim 4 \times 10^{40} \mathrm{erg} \mathrm{s}^{-1}$, where $\dot{M}_{w}=4 \pi R^{2} \rho v_{w}^{2} \Omega C$ is the outflow rate, $\Omega$ and $C$ are the solid angle and the volume filling factor (or clumpiness), respectively, which were adopted equal to 0.3 as conservative values from MHD simulations of winds driven by radiation pressure in super-Eddington winds (Takeuchi et al. 2013), $\rho$ is the density and $R$ is the distance from the ionizing source. Here we have used the $\xi$ definition to get rid of the $R^{2} \rho$ factor where $\rho=n_{\mathrm{H}} m_{p} \mu$ with $m_{p}$ the proton mass and $\mu=0.6$ the average particle weight of a highly ionized plasma.

The filling factor of the wind might be much smaller. Using equation (23) in Kobayashi et al. (2018) and assuming that the outflow rate is comparable to the accretion rate, we obtain $C \sim$ $3 \times 10^{-2}$. Systematics would tend to cancel out when also accounting for the uncertainty on the ionization parameter in the case for a harder SED $(\Delta \log \xi \sim 1)$. In the pessimistic case the wind power would be of the order of $10^{39} \mathrm{erg} \mathrm{s}^{-1}$, which means still high enough to affect the surrounding medium and inflate ISM cavities.

The spectral shape, strong wind features, and presence of dips suggest that NGC 247 ULX-1 is likely being observed at high inclination (see Fig. 12, left-hand panel) where the funnel is already obscuring the innermost, hot, hard X-ray emitting regions. As mentioned in Section 4.1, the increase of the average flux level during the intermediate observations $(3,4,5)$ might be caused by a higher local accretion rate. Such a climate change would however affect the 
properties of both the disc and the wind. The scale-height is already relevant around the Eddington limit (see, e.g.; Shakura \& Sunyaev 1973; Poutanen et al. 2007). A further increase in the local $\dot{M}$ might push the optically thick funnel further upwards (see Fig. 12, righthand panel) thereby obscuring the regions emitting photons with temperatures higher than that of the spherization radius $(\gtrsim 0.3 \mathrm{keV})$, causing the very soft dips shown in Fig. 2 (see also Urquhart \& Soria 2016).

During the dips the high-ionization portion of the wind could be hard to see as its absorption lines were primarily affecting the (now) obscured hard X-ray continuum. In fact, the strength of the highionization (1.2-1.3 keV) absorption lines clearly decreases during the dipping observations (see Fig. 4), while the $0.7 \mathrm{keV}$ O VIII absorption line seems constant in flux if not even stronger. This might also suggest a stratification in the wind. Outside the dips, an overall increase in the accretion rate would also imply a stronger radiative force and, therefore, a slightly faster wind which seems to be confirmed by the higher blueshift of the lines (see Figs 3 and 4). The $1 \mathrm{keV}$ emission lines also weaken during the bright / dipping observations, indicating that they should be produced in the inner regions in agreement with their overall larger broadening (see Table 3).

A similar picture was proposed by Guo et al. (2019) who argued that the $\sim 100$ s transitions can be explained by the viscous time-scale with the X-ray flux variability driven by accretion rate fluctuations (at $\dot{m} \gtrsim 10$ ). However, local fluctuations in the $\dot{M}$ might also cause variations in the winds, which could alter the source appearance (Feng et al. 2016).

Although fascinating and self-consistent, this scenario might be not the only one able to explain all the observables. Additional, alternative, and (ideally) model-independent approaches could be considered. For instance, another phenomenon which might explain the nature of the dips might be the propeller effect due to a strong magnetic field. Such scenario would imply a decreasing $\dot{M}$ and a geometrical beaming to cause the observed brightening. More insights on the temporal evolution of the spectral residuals accounting for the different spectral states that the source shows inside / outside the dips will be given in D'Aì et al. (in preparation). Similarly, the Fourier analysis of the characteristics time-scales in NGC 247 ULX1 and the corresponding association with the dipping activity will be shown by Alston et al. (2021). Here, in particular, we argue that the alternation of the dips might be due to azimuthally dependent structures.

We plan to investigate the variability of the RGS spectral lines to place more constraints on to their nature. However, the low count rate of the grating spectra currently prevent us from trying to study them during the dips and on time-scales shorter than $100 \mathrm{ks}$ in the bright states outside the dips. Future missions like XRISM and Athena will revolutionize the study of ULX thanks to their high effective area, high spectral resolution, and low background (see, e.g. Barret et al. 2018; Guainazzi \& Tashiro 2018). Pinto et al. (2020b) simulated NGC 1313 ULX-1 microcalorimeter spectra for these two missions and showed (1) how XRISM will strengthen the identification of lines in the 1-10 keV band and (2) how Athena / X-IFU will be able to detect winds in observations with just $1 \mathrm{ks}$ of exposure time. The latter is primarily due to the fact that X-IFU will have two orders of magnitude higher effective area than RGS.

\section{CONCLUSIONS}

Most ULXs are believed to be powered by super-Eddington accreting neutron stars and, perhaps, black holes. The disc is expected to thicken at accretion rates above the Eddington rate and to launch powerful winds through radiation pressure and/or magnetic fields. Evidence of winds has been found in several ULXs through highresolution X-ray spectrometers. It is yet unclear whether the switch between the classical soft and supersoft state - which is observed in supersoft ULXs - is due to the thickening of the disc and/or the optically thick part of the wind. In order to better understand such phenomenology and the overall super-Eddington mechanism, we undertook a large observing campaign with XMM-Newton to study NGC 247 ULX-1, which is the brightest (in flux) of all supersoft ULXs.

The new observations showed for the first time unambiguous evidence of a wind in the form of emission and absorption lines from highly ionized ionic species, with the absorption phase exhibiting a mildly relativistic outflow $(-0.17 c)$ in line with the other ULXs whose grating spectra had sufficient quality to detect and identify spectral lines. Remarkable variability was observed in the source flux with strong dipping activity during the brightest observations, which is typical among soft ULXs such as NGC 55 ULX-1, and indicate a close relationship between the accretion rate and the appearance of the dips. The latter are likely due to a thickening of the disc scale height and the wind as shown by a progressively increasing blueshift in the spectral lines.

\section{ACKNOWLEDGEMENTS}

This work is based on observations obtained with XMM-Newton, an ESA science mission funded by ESA Member States and USA (NASA). We acknowledge support from ESA Research Fellowships. We thank the XMM-Newton SOC for support in optimizing our observing campaign and J. M. Miller, D. Proga, and M. Parker for useful discussion regarding winds and absorption in Galactic Xray binary. $\mathrm{AD}, \mathrm{MDS}$, EA acknowledge financial support from the agreement Italian Space Agency (ASI) - INAF no. 2017-14-H.0 and INAF main-stream. We thank the anonymous referee for their very useful suggestions.

\section{DATA AVAILABILITY}

All of the data and software used in this work are publicly available from ESA's XMM-Newton Science Archive (XSA ${ }^{1}$ ) and NASA's HEASARC archive. ${ }^{2}$ Our codes are publicly available and can be found on the GitHub. ${ }^{3}$

\section{REFERENCES}

Allevato V., Paolillo M., Papadakis I., Pinto C., 2013, ApJ, 771, 9

Alston W. N. et al., 2021, MNRAS, 505, 3722

Bachetti M. et al., 2014, Nature, 514, 202

Barret D. et al., 2018, in den Herder J.-W. A., Nikzad S., Nakazawa K., eds, Proc. SPIE Conf. Ser. Vol. 10699, The ATHENA X-ray Integral Field Unit (X-IFU). SPIE, Bellingham, p. 106991G

Begelman M. C., King A. R., Pringle J. E., 2006, MNRAS, 370, 399

Belfiore A. et al., 2020, Nat. Astron., 4, 147

Brightman M. et al., 2016, ApJ, 816, 60

Brinkmann W., Aschenbach B., Kawai N., 1996, A\&A, 312, 306

Cash W., 1979, ApJ, 228, 939

Earnshaw H. M., Roberts T. P., 2017, MNRAS, 467, 2690

\footnotetext{
${ }^{1}$ https://www.cosmos.esa.int/web/xmm-newton/xsa

${ }^{2}$ https://heasarc.gsfc.nasa.gov/

${ }^{3}$ https://github.com/ciropinto1982
} 
El Mellah I., Casse F., 2017, MNRAS, 467, 2585

Fabrika S., 2004, Astrophys. Space Phys. Rev., 12, 1

Feng H., Tao L., Kaaret P., Grisé F., 2016, ApJ, 831, 117

Gladstone J. C., Roberts T. P., Done C., 2009, MNRAS, 397, 1836

Goad M. R., Roberts T. P., Reeves J. N., Uttley P., 2006, MNRAS, 365, 191

Guainazzi M., Tashiro M. S., 2018, preprint (arXiv:1807.06903)

Guo J., Sun M., Gu W.-M., Yi T., 2019, MNRAS, 485, 2558

Gúrpide A., Godet O., Koliopanos F., Webb N., Olive J.-F., 2021, A\&A, 649, A104

Kaaret P., Feng H., Roberts T. P., 2017, ARA\&A, 55, 303

Kaastra J. S., 2017, A\&A, 605, A51

Kaastra J. S., Mewe R., Nieuwenhuijzen H., 1996, in Yamashita K., Watanabe T., eds, UV and X-ray Spectroscopy of Astrophysical and Laboratory Plasmas, SPEX: a New Code for Spectral Analysis of X \& UV Spectra. p. 411

King A., Lasota J.-P., 2020, MNRAS, 494, 3611

King A. R., Davies M. B., Ward M. J., Fabbiano G., Elvis M., 2001, ApJ, 552, L109

Kobayashi H., Ohsuga K., Takahashi H. R., Kawashima T., Asahina Y., Takeuchi S., Mineshige S., 2018, PASJ, 70, 22

Koliopanos F., Vasilopoulos G., Godet O., Bachetti M., Webb N. A., Barret D., 2017, A\&A, 608, A47

Kosec P., Pinto C., Fabian A. C., Walton D. J., 2018a, MNRAS, 473, 5680

Kosec P., Pinto C., Walton D. J., Fabian A. C., Bachetti M., Brightman M., Fürst F., Grefenstette B. W., 2018b, MNRAS, 479, 3978

Kosec P., Zoghbi A., Walton D. J., Pinto C., Fabian A. C., Parker M. L., Reynolds C. S., 2020, MNRAS, 495, 4769

Krautter J., Oegelman H., Starrfield S., Wichmann R., Pfeffermann E., 1996, ApJ, 456, 788

Krolik J. H., McKee C. F., Tarter C. B., 1981, ApJ, 249, 422

Liu J.-F., 2008, ApJS, 177, 181

Marshall H. L., Canizares C. R., Schulz N. S., 2002, ApJ, 564, 941

Marshall H. L., Canizares C. R., Hillwig T., Mioduszewski A., Rupen M., Schulz N. S., Nowak M.,Heinz S., 2013, ApJ, 775, 75

Medvedev P. S., Khabibullin I. I., Sazonov S. Y., Churazov E. M., Tsygankov S. S., 2018, Astron. Lett., 44, 390

Medvedev P. S., Khabibullin I. I., Sazonov S. Y., 2019, Astron. Lett., 45, 299

Middleton M. J., Roberts T. P., Done C., Jackson F. E., 2011, MNRAS, 411, 644

Middleton M. J., Heil L., Pintore F., Walton D. J., Roberts T. P., 2015a, MNRAS, 447, 3243

Middleton M. J., Walton D. J., Fabian A., Roberts T. P., Heil L., Pinto C., Anderson G., Sutton A., 2015b, MNRAS, 454, 3134

Middleton M. J. et al., 2018, preprint (arXiv:1810.10518)

Miller J. M., Cackett E. M., Reis R. C., 2009, ApJ, 707, L77

Nandra K., George I. M., Mushotzky R. F., Turner T. J., Yaqoob T., 1997, ApJ, 476, 70

Pinto C., Ness J.-U., Verbunt F., Kaastra J. S., Costantini E., Detmers R. G., 2012, A\&A, 543, A134

Pinto C., Kaastra J. S., Costantini E., de Vries C., 2013, A\&A, 551, A25

Pinto C., Middleton M. J., Fabian A. C., 2016, Nature, 533, 64

Pinto C. et al., 2017, MNRAS, 468, 2865

Pinto C. et al., 2020a, MNRAS, 491, 5702

Pinto C. et al., 2020b, MNRAS, 492, 4646

Pintore F., Esposito P., Zampieri L., Motta S., Wolter A., 2015, MNRAS, 448, 1153

Poutanen J., Lipunova G., Fabrika S., Butkevich A. G., Abolmasov P., 2007, MNRAS, 377, 1187

Psaradaki I., Costantini E., Mehdipour M., Díaz Trigo M., 2018, A\&A, 620, A129

Qiu Y., Feng H., 2021, ApJ, 906, 36

Shakura N. I., Sunyaev R. A., 1973, A\&A, 24, 337

Soria R., Motch C., Read A. M., Stevens I. R., 2004, A\&A, 423, 955

Stobbart A.-M., Roberts T. P., Wilms J., 2006, MNRAS, 368, 397

Sutton A. D., Roberts T. P., Middleton M. J., 2013, MNRAS, 435, 1758

Takeuchi S., Ohsuga K., Mineshige S., 2013, PASJ, 65, 88

Tarter C. B., Tucker W. H., Salpeter E. E., 1969, ApJ, 156, 943

Urquhart R., Soria R., 2016, MNRAS, 456, 1859
Vaughan S., Edelson R., Warwick R. S., Uttley P., 2003, MNRAS, 345, 1271

Walton D. J. et al., 2014, ApJ, 793, 21

Walton D. J. et al., 2017, ApJ, 839, 110

Walton D. J. et al., 2018a, ApJ, 856, 128

Walton D. J. et al., 2018c, MNRAS, 473, 4360

Wang C., Soria R., Wang J., 2019, ApJ, 883, 44

\section{APPENDIX A: TECHNICAL DETAILS}

In this section we put technical detail, plots, and tables that were excluded from the main body of the paper.

\section{A1 Nearby bright X-ray source}

The RGS extraction region includes a few faint sources with the brightest one being XMMU J004710.0-204708 (X-2 hereafter, see Fig. 1). We extracted its EPIC spectra from all observations and stacked them similarly to ULX-1. The spectrum of X-2 is much flatter than that of the ULX-1 and can be well modelled with a powerlaw model $(\Gamma=1.60 \pm 0.03)$, a moderate column density $N_{\mathrm{H}}=$ $(1.0 \pm 0.1) \times 10^{21} \mathrm{~cm}^{-2}$, and an intrinsic luminosity $L_{0.3-10 \mathrm{keV}}=$ $(1.45 \pm 0.04) \times 10^{38} \mathrm{erg} \mathrm{s}^{-1}$ (assuming a distance of $3.3 \mathrm{Mpc}$ ). This corresponds to the Eddington limit for a Solar-mass star and, given the spectral slope, the source X-2 could be a common XRB near the NGC 247 centre. At $1 \mathrm{keV}$ its spectrum is remarkably featureless and 40-50 times fainter than ULX-1 implying that it will have no significant effects on the RGS spectral lines.

\section{A2 Modelling of individual EPIC observations}

Table A1 shows the results of the EPIC spectral modelling and the RMS estimated from the EPIC-pn data of each observation (see Sections 3.1 and 2.1.4).

\section{A3 CIE model scan for the SS 433 RGS spectrum}

We analysed the XMM-Newton RGS spectrum of SS 433 from the observation id:0694870201 (2012-10-03), which provides the longest $(\sim 130 \mathrm{ks})$ and best-exposed RGS spectrum of SS 433 . We reduced the RGS spectrum of SS 433 obsid 0694870201 identically to that of the NGC 247 ULX-1 data shown in Section 2.1.2. After removing the very little Solar flares we are left with $129.4 \mathrm{ks}$ for both RGS 1 and 2 cameras. We used the 6-25 A range because at higher wavelengths the source emission is significantly absorbed and the background noise dominates the RGS spectrum (see Fig. A1). No significant pile up was found in the RGS spectra.

Strong emission lines were observed close to the rest-frame energies of the most relevant transitions such as Si XIII, Mg XI, Ne X, Ne IX, Fe XVII, and O VIII, which is very similar to NGC 247 ULX-1, albeit at higher significance because SS 433 is much closer ( $\sim \mathrm{kpc})$ and brighter. We modelled the RGS spectral continuum with an absorbed power-law model obtaining results similar to Marshall et al. (2013) and Medvedev et al. (2018) such as a column density $N_{\mathrm{H}}=$ $(1.14 \pm 0.02) \times 10^{22} \mathrm{~cm}^{-2}$, a slope $\Gamma=2.53 \pm 0.06$ and an X-ray unabsorbed luminosity $L_{[0.3-10 \mathrm{keV}]}=(1.03 \pm 0.05) \times 10^{36} \mathrm{erg} \mathrm{s}^{-1}$. We obtained high C-stat/d.o.f $=2393 / 618$ as expected, due to the strong, unmodelled, emission lines.

We tested on to the SS 433 RGS spectrum the same routine used for the NGC 247 ULX-1 data in Section 3.4.2 to check the robustness of our method. We adopted collisional ionization equilibrium (cie model in SPEX) to model the jet emission. The velocity dispersion was fixed to $500 \mathrm{~km} \mathrm{~s}^{-1}$, i.e. close to the RGS spectral resolution, 


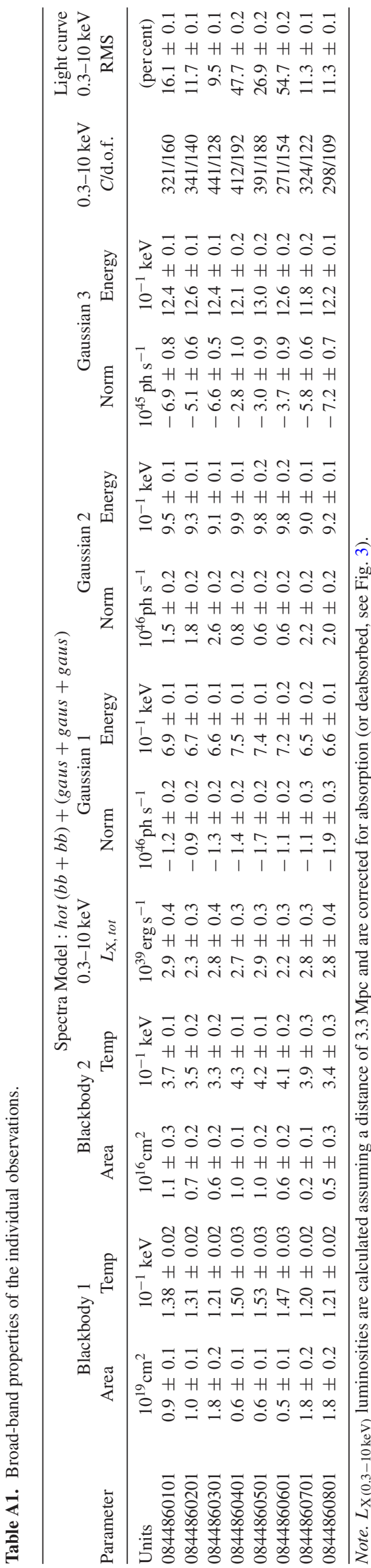

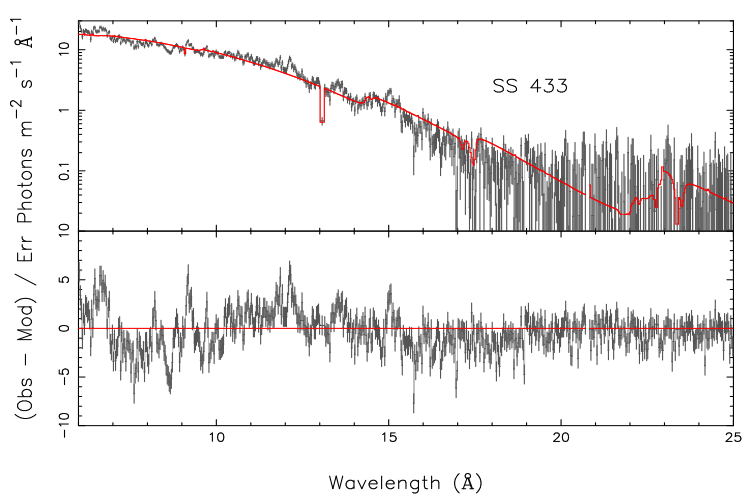

Figure A1. SS 433 RGS spectrum and continuum model.

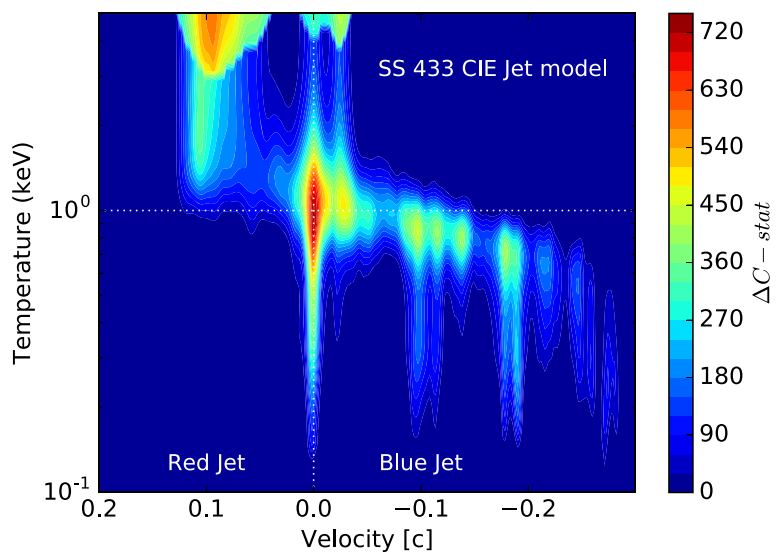

Figure A2. Multidimensional scan of collisional-ionization emission model for the SS 433 RGS spectrum. The X-axis shows the line-of-sight velocity. Labels are the same as in Fig. 9.

the abundances were chosen to be Solar (to limit the computing time). We found a dominant low-velocity solution with an average temperature of $1 \mathrm{keV}$ (see dotted horizontal and vertical lines in Fig. A2). The velocity is consistent with the dynamical range found by Medvedev et al. (2018) using the Fe K lines from the EPIC-pn spectrum, but showed a lower temperature, which is expected given that the RGS spectrum is more sensitive to the cooler gas phase of the multitemperature jet.

\section{A4 SED modelling and systematics effects}

The non-detection of the optical and UV counterpart of NGC 247 ULX-1 (Section 2.1.3) prevent us from building a simultaneous multiwavelength SED for our source. This might have systematic effects on the calculation of the photoionization balance. Moreover, the flux upper limits obtained with the OM suggest that at least in the far-UV energy band the source flux was lower than the levels measured in the archival $H S T$ observations that we used here.

Pinto et al. (2020a) showed that a lower IR-to-UV flux in moderately hard sources, such as NGC 1313 ULX-1, mainly strengthens thermal instabilities at intermediate temperatures and ionization parameters. In Fig. A3, we show the SED adopted here (solid black curve) consisting of a five-blackbody model along with the simple 2blackbody model (dashed-dotted black line, see also Section 3.5.1). The lower panel shows the stability curves computed for these models. Some deviations are mainly seen at $\log \xi$ from $1.5-2.5$, which 

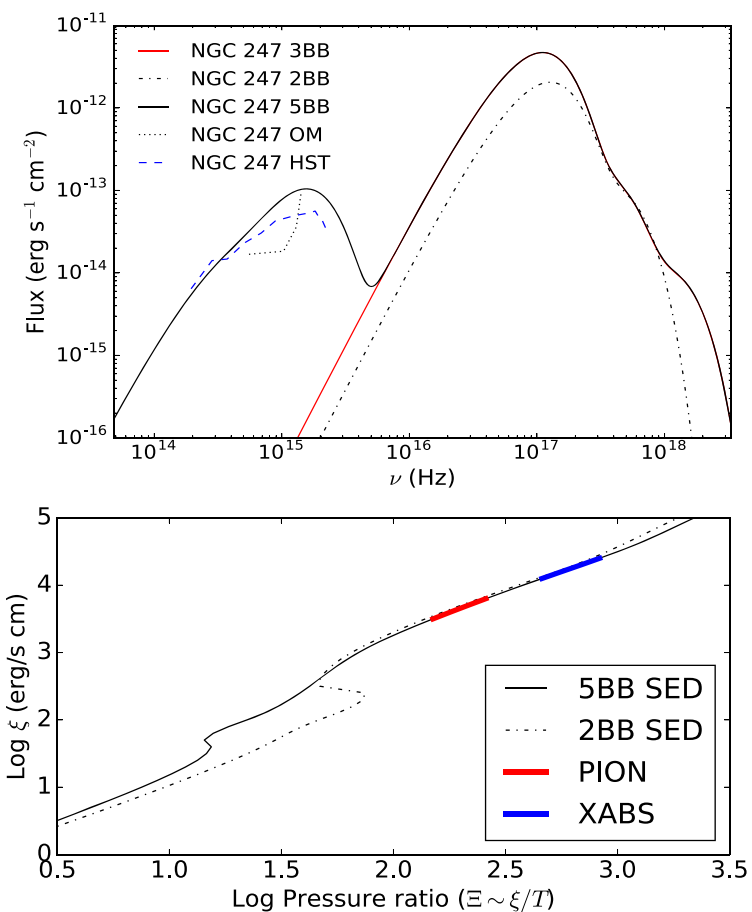

Figure A3. SED (top panel) and thermal-stability curve (bottom panel) computed for the time-averaged spectrum of NGC 247 ULX-1. The baseline SED (solid black line) consists of five unabsorbed blackbody models that account for emission in the optical, UV, and X-ray bands. An alternative, simpler SED model uses just the X-ray two-blackbody model (dashed-dotted line).

is well below the values measured in this work (see Table 3). This is not surprising given the shortage of hard X-ray in our spectra which are the primary responsible of thermal instabilities. This suggests that the ionization balance is not significantly affected even if the optical / UV fluxes were 2 orders of magnitude lower than our assumptions.

\section{A5 Monte Carlo simulations and significance}

The $\Delta C_{\max }$ improvement to the continuum model does not necessarily yield the significance of the corresponding emission- or absorption-line models. This is due to the large parameters space that was explored and the possibility of detecting random spectral features (the look-elsewhere effect).

Among our physical model searches, the one for the absorption lines provided the smallest $\Delta C_{\max }$ due to their strength being lower than that typical of the emission lines. We therefore focused on the results obtained with the xabs component and used them as a proxy for the pion.

Following the method used in Pinto et al. (2020b), we simulated 20000 RGS and EPIC spectra adopting the 3-blackbody continuum model. Each faked spectrum was scanned with the same $x a b s$ grids used in Section 3.5.3. The results of our MC simulations are shown

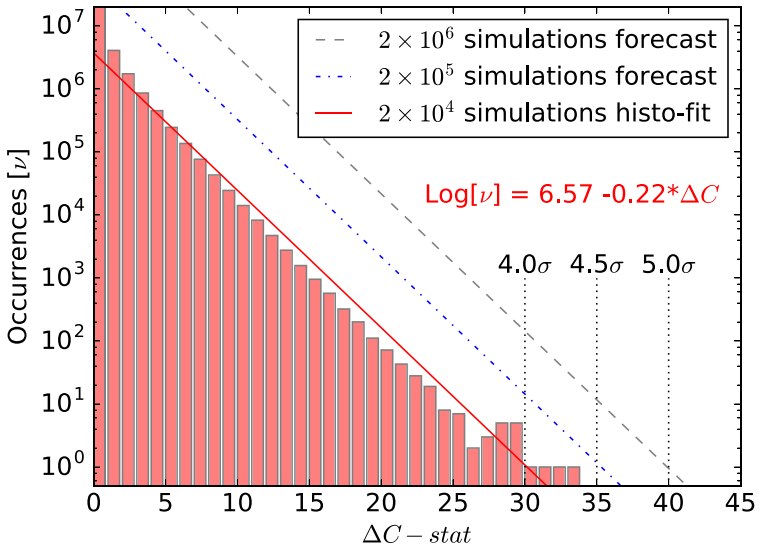

Figure A4. Histogram and corresponding power-law fit of the $20 \mathrm{k}$ Monte Carlo simulations of NGC 247 ULX-1 and forecast for 200000 and 2 million simulations.

in Fig. A4. No outlier was found with $\Delta C \geq \Delta C_{\max }=46$, which suggests a significance $>4 \sigma$ for the absorbing gas.

We compared the simulations histogram for NGC 247 ULX-1 with those obtained for different sources by adopting a similar approach: $20 \mathrm{k}$ simulations of NGC 1313 ULX-1 (Pinto et al. 2020b), $2 \mathrm{k}$ for NGC 5204 ULX-1 (Kosec et al. 2018a) and $50 \mathrm{k}$ for the same data with a new, faster, cross-correlation method (Kosec et al. submitted), $20 \mathrm{k}$ for ULX NGC 7793 P13 (Pinto et al. in prepation), and $1 \mathrm{k}$ for AGN PG 1448 (Kosec et al. 2020). We fit the histograms of the logarithm of the occurrences with straight lines and found an average slope $\bar{\Gamma}=-0.225 \pm 0.015$, which agrees with the simulations of NGC 247 ULX-1 $(\Gamma=-0.218 \pm 0.006)$.

We used the best-fitting straight lines to estimate the overall shape of the $\Delta C$-stat distribution and forecast the results of larger numbers of simulations thanks to the agreement between the trends from 1000 to 50000 simulations. We therefore scaled the histogram fit of NGC 247 ULX-1, assuming a constant slope and multiplying the intercept of the straight line by a number equal to the ratio of the parameter space that we want to forecast for a given number of simulations and the one we obtained with 20000 simulations. In Fig. A4, we show the predictions for $2 \times 10^{5}$ (dash-dotted line) and $2 \times 10^{6}$ (dashed line) simulations. This would suggest 4.5 and $5 \sigma$ detection probabilities for $\Delta C$-stat above 35 and 40, respectively, in the data with an uncertainty of $0.2 \sigma$ according to the spread in the slope of the other histograms.

We finally retrieved the various $\Delta C$-stat values that correspond to confidence levels ranging from 2.0, 2.5, .., $5.0 \sigma$ and plot them as black contours in Fig. 10. The $\sigma$ contours for the pion model scan were calculated by scaling the parameter space in the histogram of the xabs simulations in the same way used for the forecast.

This paper has been typeset from a $\mathrm{T}_{\mathrm{E}} \mathrm{X} / \mathrm{L} \mathrm{T}_{\mathrm{E}} \mathrm{X}$ file prepared by the author. 